\title{
A Zonotope and a Product of Two Simplices with Disconnected Flip Graphs
}

\author{
Gaku Liu' $^{1}$
}

Received: 19 May 2016 / Revised: 8 January 2018 / Accepted: 6 February 2018 /

Published online: 19 March 2018

(C) The Author(s) 2018

\begin{abstract}
We give an example of a three-dimensional zonotope whose set of tight zonotopal tilings is not connected by flips. Using this, we show that the set of triangulations of $\Delta^{4} \times \Delta^{n}$ is not connected by flips for large $n$. Our proof makes use of a non-explicit probabilistic construction.
\end{abstract}

Keywords Zonotope $\cdot$ Zonotopal tiling $\cdot$ Flip graph $\cdot$ Product of two simplices

\section{Mathematics Subject Classification 52B11}

\section{Introduction}

We consider the poset $\mathcal{P}$ of polyhedral subdivisions of a polytope $P$ or zonotopal tilings of a zonotope $Z$, ordered by refinement. This poset is called the Baues poset of $P$ or $Z$. The minimal elements of this poset are, respectively, the triangulations of $P$ or the tight zonotopal tilings of $Z$. Two minimal elements of $\mathcal{P}$ are connected by a flip if there is an element of $\mathcal{P}$ whose only proper refinements are these two minimal elements. The flip graph of $P$ or $Z$ is the graph whose vertices are minimal elements of $\mathcal{P}$ and whose edges are flips.

We are concerned in this paper with connectivity of the flip graph. For zonotopal tilings, the flip graph is known to be connected for cyclic zonotopes [19] or if the zonotope has dimension two [5]. Our first result is the first example of a zonotope whose

Editor in Charge: Kenneth Clarkson

Gaku Liu

gakuliu@gmail.com

1 Max Planck Institute for Mathematics in the Sciences, 04103 Leipzig, Germany 
flip graph is not connected. This answers a question of Reiner in [9]. Our example is a three-dimensional permutohedron with many copies of each of its generating vectors. The number of copies of each vector is determined by a probabilistic argument to be around 100 .

Using a related construction, we also show that the flip graph of triangulations of the product of two simplices is not generally connected. Santos [10] gave the first example of a polytope whose flip graph of triangulations is not connected. However, the case when $P$ is a product of two simplices remained of special interest due to the appearances of these triangulations in various branches of mathematics; see [2, Chap. 6.2] for an overview. Santos [14] proved that the flip graph of $\Delta^{2} \times \Delta^{n}$ is connected for all $n$, and the author [6] proved that the flip graph of $\Delta^{3} \times \Delta^{n}$ is connected for all $n$. However, we show that the flip graph of $\Delta^{4} \times \Delta^{n}$ is not connected for $n \approx 4 \cdot 10^{4}$.

Triangulations of lattice polytopes are closely related to toric varieties in algebraic geometry. Each lattice polytope defines a toric ideal and an associated toric Hilbert scheme. For a totally unimodular polytope, the associated toric Hilbert scheme is connected if and only if the flip graph of the polytope is connected; see [17, Chap. 10] or [7]. Thus, our result implies that the toric Hilbert scheme of $\Delta^{4} \times \Delta^{n}$ is not connected for large $n$. While non-connected toric Hilbert schemes had previously been constructed [13], our proof demonstrates non-connectivity for the first time for a totally unimodular polytope. In addition, the toric ideal associated to $\Delta^{m} \times \Delta^{n}$ is the well-studied determinantal ideal generated by $2 \times 2$ minors of an $(m+1) \times(n+1)$ matrix; its zero-locus is the Segre variety.

Connectivity of flip graphs is also related to the generalized Baues problem, formulated by Billera et al. [1], which concerns the topology of the Baues poset $\mathcal{P}$. Specifically, the problem asks if the order complex of $\mathcal{P}$ minus its maximal element is homotopy equivalent to a sphere. While the problem has been resolved for most cases of interest, it remains open for zonotopes, and is of particular interest in this case because it is equivalent to the extension space conjecture for oriented matroids, which states that the extension space of a realizable oriented matroid is homotopy equivalent to a sphere. See $[11,18]$ for definitions and known results. In general, the generalized Baues problem and the question of flip graph connectivity do not imply each other. However, there are situations where the answer to one can be used to answer the other. The generalized Baues problem for triangulations was answered in the negative by Santos [15] by constructing a point set in general position with disconnected flip graph. The problem for zonotopal tilings remains open, but would be answered in the negative if a zonotope with generating vectors in general position was found to have disconnected flip graph; see [9, Lem. 3.1].

The paper is organized as follows. Section 2 reviews triangulations and the product of two simplices. Section 3 reviews mixed subdivisions, zonotopal tilings, and the Cayley trick. Section 4 constructs our zonotope and proves that its flip graph is not connected. Section 5 proves that the flip graph of $\Delta^{4} \times \Delta^{n}$ is not connected. Section 6 is an appendix proving several propositions used in the paper. 


\section{Triangulations and the Product of Two Simplices}

We begin with a quick overview of triangulations, flips, and the product of two simplices. We refer to De Loera et al. [2] for a more comprehensive treatment.

\subsection{Subdivisions and Triangulations}

Throughout this section, let $A \subset \mathbb{R}^{m}$ be a finite set of points. A cell of $A$ is a subset of $A$. A simplex is a cell which is affinely independent. A face of a cell $C$ is a subset $F \subseteq C$ such that there exists a linear functional $\phi \in\left(\mathbb{R}^{m}\right)^{*}$ such that $F$ is the set of all points which minimize $\phi$ on $C$. For any cell $C$, let $\operatorname{conv}(C)$ denote the convex hull of $C$.

Definition 2.1 A polyhedral subdivision, or subdivision, of $A$ is a collection $\mathscr{S}$ of cells of $A$ such that

1. If $C \in \mathscr{S}$ and $F$ is a face of $C$, then $F \in \mathscr{S}$.

2. If $C, C^{\prime} \in \mathscr{S}$, then $\operatorname{conv}(C) \cap \operatorname{conv}\left(C^{\prime}\right)=\operatorname{conv}(F)$ where $F$ is a face of $C$ and $C^{\prime}$.

3. $\bigcup_{C \in \mathscr{S}} \operatorname{conv}(C)=\operatorname{conv}(A)$.

The subdivision consisting of $A$ and all faces of $A$ is the trivial subdivision. A subdivision all of whose elements are simplices is a triangulation.

If $\mathscr{S}$ is a subdivision of $A$ and $F$ is a face of $A$, then $\mathscr{S}$ induces a subdivision $\mathscr{S}[F]$ of $F$ by

$$
\mathscr{S}[F]:=\{C \in \mathscr{S}: C \subseteq F\} .
$$

For subdivisions $\mathscr{S}, \mathscr{S}^{\prime}$, we say that $\mathscr{S}$ is a refinement of $\mathscr{S}^{\prime}$ if every element of $\mathscr{S}$ is a subset of an element of $\mathscr{S}^{\prime}$. Refinement gives a poset structure on the set of all subdivisions of $A$. The maximal element of this poset is the trivial subdivision and the minimal elements are the triangulations.

\subsection{Flips}

As stated in the introduction, two triangulations are connected by a flip if there is a subdivision whose only proper refinements are these two triangulations. We will now give an equivalent definition of a flip which will be easier to use.

A circuit is a minimal affinely dependent subset of $\mathbb{R}^{m}$. If $X=\left\{x_{1}, \ldots, x_{k}\right\}$ is a circuit, then the elements of $X$ satisfy an affine dependence equation

$$
\sum_{i=1}^{k} \lambda_{i} x_{i}=0
$$

where $\lambda_{i} \in \mathbb{R} \backslash\{0\}$ for all $i, \sum_{i} \lambda_{i}=0$, and the equation is unique up to multiplication by a constant. This gives a unique partition $X=X^{+} \cup X^{-}$of $X$ given by $X^{+}=\left\{x_{i}\right.$ : 
$\left.\lambda_{i}>0\right\}$ and $X^{-}=\left\{x_{i}: \lambda_{i}<0\right\}$. We will write $X=\left(X^{+}, X^{-}\right)$to denote a choice of which part we call $X^{+}$and which we call $X^{-}$.

A circuit $X=\left(X^{+}, X^{-}\right)$has exactly two non-trivial subdivisions, which are the following triangulations:

$$
\mathscr{T}_{X}^{+}:=\left\{\sigma \subseteq X: \sigma \nsupseteq X^{+}\right\}, \quad \mathscr{T}_{X}^{-}:=\left\{\sigma \subseteq X: \sigma \nsupseteq X^{-}\right\} .
$$

Given a subdivision $\mathscr{S}$ and a cell $C \in \mathscr{S}$, we define the link of $C$ in $\mathscr{S}$ as

$$
\operatorname{link}_{\mathscr{S}}(C):=\left\{C^{\prime} \in \mathscr{S}: C \cap C^{\prime}=\emptyset, C \cup C^{\prime} \in \mathscr{S}\right\}
$$

We can now state the definition of a flip, in the form of a proposition.

Proposition 2.2 (Santos [12]) Let $\mathscr{T}$ be a triangulation of A. Suppose there is a circuit $X=\left(X^{+}, X^{-}\right)$contained in A such that

1. $\mathscr{T}_{X}^{+} \subseteq \mathscr{T}$.

2. All maximal simplices of $\mathscr{T}_{X}^{+}$have the same link $\mathscr{L}$ in $\mathscr{T}$.

Then the collection

$$
\mathscr{T}^{\prime}:=\mathscr{T} \backslash\left\{\rho \cup \sigma: \rho \in \mathscr{L}, \sigma \in \mathscr{T}_{X}^{+}\right\} \cup\left\{\rho \cup \sigma: \rho \in \mathscr{L}, \sigma \in \mathscr{T}_{X}^{-}\right\}
$$

is a triangulation of $A$. We say that $\mathscr{T}$ has a flip supported on $\left(X^{+}, X^{-}\right)$, and that $\mathscr{T}^{\prime}$ is the result of applying this flip to $\mathscr{T}$. The flip graph (on triangulations) of $\mathscr{T}$ is the graph whose vertices are triangulations of $\mathscr{T}$ and with an edge between two triangulations if one is obtained from the other by a flip.

The following fact is a convenient way to determine whether a flip supported on a certain circuit exists. It is proved in [6].

Proposition 2.3 Let $\mathscr{T}$ be a triangulation of $A$ and let $X=\left(X^{+}, X^{-}\right)$be a circuit in A. Suppose that $X^{-} \in \mathscr{T}$. Then $\mathscr{T}$ has a flip supported on $\left(X^{+}, X^{-}\right)$if and only if there is no maximal simplex $\tau \in \mathscr{T}$ with $X^{-} \subseteq \tau$ and $|X \cap \tau| \leq|X|-2$.

\subsection{Regular Subdivisions}

Let $A \subseteq \mathbb{R}^{m}$ be as before. Let $\omega: A \rightarrow \mathbb{R}$ be any function. For a cell $C \subseteq A$, we define the lift of $C$ to be the set $C^{\omega} \subset \mathbb{R}^{m} \times \mathbb{R}$ given by

$$
C^{\omega}:=\{(x, \omega(x)): x \in C\} .
$$

We call a subset $F \subseteq A^{\omega}$ a lower face of $A^{\omega}$ if either $F$ is empty or there is a linear functional $\phi \in\left(\mathbb{R}^{m} \times \mathbb{R}\right)^{*}$ such that $\phi(0,1)>0$ and $F$ is the set of all points which minimize $\phi$ on $A^{\omega}$. Then the collection of all $C \subseteq A$ such that $C^{\omega}$ is a lower face of $A^{\omega}$ is a subdivision of $A$. We call this the regular subdivision of $A$ with respect to $\omega$, and denote it by $\mathscr{S}_{A}^{\omega}$.

If $F$ is a face of $A$, then $\mathscr{S}_{A}^{\omega}[F]=\mathscr{S}_{F}^{\left.\omega\right|_{F}}$.

Both triangulations of a circuit are regular, as stated below. 
Proposition 2.4 Suppose $\sum_{i=1}^{k} \lambda_{i} x_{i}=0$ is the affine dependence equation for $a$ circuit $X=\left(X^{+}, X^{-}\right)$with $X^{+}=\left\{x_{i}: \lambda_{i}>0\right\}$ and $X^{-}=\left\{x_{i}: \lambda_{i}<0\right\}$. Let $\omega: X \rightarrow \mathbb{R}$ be a function. Then

$$
\mathscr{S}_{X}^{\omega}= \begin{cases}\mathscr{T}_{X}^{+} & \text {if } \sum_{i=1}^{k} \lambda_{i} \omega\left(x_{i}\right)>0 \\ \mathscr{T}_{X}^{-} & \text {if } \sum_{i=1}^{k} \lambda_{i} \omega\left(x_{i}\right)<0\end{cases}
$$

\subsection{The Product of Two Simplices}

We now consider $\Delta^{m-1} \times \Delta^{n-1}$, the product of two simplices of dimensions $m-1$ and $n-1$. Following the conventions of the previous section, we will understand $\Delta^{m-1} \times \Delta^{n-1}$ to mean the set of vertices of $\Delta^{m-1} \times \Delta^{n-1}$ rather than the polytope itself.

Let $\Delta^{m-1}:=\left\{e_{1}, \ldots, e_{m}\right\}$ be the standard basis for $\mathbb{R}^{m}$ and $\Delta^{n-1}:=\left\{f_{1}, \ldots, f_{n}\right\}$ be the standard basis for $\mathbb{R}^{n}$. We embed $\Delta^{m-1} \times \Delta^{n-1}$ in $\mathbb{R}^{m} \times \mathbb{R}^{n}$ by

$$
\Delta^{m-1} \times \Delta^{n-1}:=\left\{\left(e_{i}, f_{j}\right): i \in[m], j \in[n]\right\} .
$$

Let $G:=K_{m, n}$ be the complete bipartite graph with vertex set $\Delta^{m-1} \cup \Delta^{n-1}$ and edge set $\left\{e_{i} f_{j}: i \in[m], j \in[n]\right\}$. We have a bijection $\left(e_{i}, f_{j}\right) \mapsto e_{i} f_{j}$ between $\Delta^{m-1} \cup \Delta^{n-1}$ and the edge set of $G$. For each cell $C \subseteq \Delta^{m-1} \times \Delta^{n-1}$, let $G(C)$ be the minimal subgraph of $G$ with edge set $\left\{e_{i} f_{j}:\left(e_{i}, f_{j}\right) \in C\right\}$. Then $C$ is a simplex if and only if $G(C)$ is acyclic, and $C$ is a circuit if and only if $G(C)$ is a cycle. If $C$ is a circuit, then $G(C)$ alternates between edges corresponding to positive and negative elements of the circuit.

\section{Zonotopal Tilings}

We now define zonotopal tilings in terms of mixed subdivisions. We review the Cayley trick which shows that mixed subdivisions can be thought of as polyhedral subdivisions of another polytope. The information in this section was developed in [4], [14], and [16].

\subsection{Mixed Subdivisions}

Let $A_{1}, \ldots, A_{n}$ be finite subsets of $\mathbb{R}^{m}$. The Minkowski sum of $A_{1}, \ldots, A_{n}$ is the set of points

$$
\sum A_{i}=A_{1}+\cdots+A_{n}:=\left\{x_{1}+\cdots+x_{n}: x_{i} \in A_{i} \text { for all } i\right\} .
$$

In this paper, we want the phrase " $\sum A_{i}$ " to identify a set of points but also retain the information of what $A_{1}, \ldots, A_{n}$ are. In other words, $\sum A_{i}$ will formally mean an ordered tuple $\left(A_{1}, \ldots, A_{n}\right)$ but by abuse of notation will also refer to the Minkowski sum. 
A mixed cell of $\sum A_{i}$ is a set $\sum B_{i}$ where $B_{i}$ is a cell of $A_{i}$ for all $i$. A mixed cell is fine if all the $B_{i}$ are simplices and lie in independent affine subspaces. A face of a mixed cell $\sum B_{i}$ is a mixed cell $\sum F_{i}$ of $\sum B_{i}$ such that there exists a linear functional $\phi \in\left(\mathbb{R}^{m}\right)^{*}$ such that for all $i$, either $F_{i}=\emptyset$ or $F_{i}$ is the set of all points which minimize $\phi$ on $B_{i}$.

Definition 3.1 A mixed subdivision of $\sum A_{i}$ is a collection $\mathscr{S}$ of mixed cells of $\sum A_{i}$ such that

1. If $\sum B_{i} \in \mathscr{S}$ and $\sum F_{i}$ is a face of $\sum B_{i}$, then $\sum F_{i} \in \mathscr{S}$.

2. If $\sum B_{i}, \sum B_{i}^{\prime} \in \mathscr{S}$, then $\operatorname{conv}\left(\sum B_{i}\right) \cap \operatorname{conv}\left(\sum B_{i}^{\prime}\right)=\operatorname{conv}\left(\sum F_{i}\right)$ where $\sum F_{i}$ is a face of $\sum B_{i}$ and $\sum B_{i}^{\prime}$.

3. $\bigcup_{\sum B_{i} \in \mathscr{S}} \operatorname{conv}\left(\sum B_{i}\right)=\operatorname{conv}\left(\sum A_{i}\right)$.

A mixed subdivision is fine if all of its elements are fine.

For mixed subdivisions $\mathscr{S}, \mathscr{S}^{\prime}$, we say that $\mathscr{S}$ is a refinement of $\mathscr{S}^{\prime}$ if every element of $\mathscr{S}$ is a mixed cell of an element of $\mathscr{S}^{\prime}$. Refinement gives a poset structure on the set of mixed subdivisions of $\sum A_{i}$ whose minimal elements are the fine mixed subdivisions.

\subsection{Basic Sums and Zonotopes}

Let $A_{1}, \ldots, A_{n}$ be as in the previous section. Let $\Delta^{m-1}=\left\{e_{1}, \ldots, e_{m}\right\}$ be the standard basis of $\mathbb{R}^{m}$. We will say that $\sum A_{i}$ is basic if $A_{i} \subseteq \Delta^{m-1}$ for all $i$.

Let $A_{1}, \ldots, A_{n}$ be as in the previous section, and assume additionally that $\left|A_{i}\right|=$ 2 for all $i$. Then $\sum A_{i}$ is called a zonotope, and its mixed subdivisions are called zonotopal tilings. A fine zonotopal tiling is called a tight zonotopal tiling.

Example 3.2 For all $1 \leq i<j \leq m$, let $A_{i j}=\left\{e_{i}, e_{j}\right\}$. Then $\Pi^{m-1}:=\sum_{1 \leq i<j \leq m} A_{i j}$ is a basic zonotope called the $(m-1)$-dimensional permutohedron.

\subsection{Coherent Mixed Subdivisions}

Let $A_{1}, \ldots, A_{n}$ be finite subsets of $\mathbb{R}^{m}$. For each $i=1, \ldots, n$, let $\omega_{i}: A_{i} \rightarrow \mathbb{R}$ be a function. For a mixed cell $\sum B_{i}$ of $\sum A_{i}$, define the lift $\left(\sum B_{i}\right)^{\omega}$ by

$$
\left(\sum B_{i}\right)^{\omega}:=\sum B_{i}^{\omega_{i}}
$$

We call a mixed cell $\sum F_{i}$ of $\left(\sum A_{i}\right)^{\omega}$ a lower face of $\left(\sum A_{i}\right)^{\omega}$ if there is a linear functional $\phi \in\left(\mathbb{R}^{m} \times \mathbb{R}\right)^{*}$ such that $\phi(0,1)>0$ and for each $i$, either $F_{i}$ is empty or $F_{i}$ is the set of points which minimize $\phi$ on $A_{i}^{\omega_{i}}$. The collection of all $\sum B_{i}$ such that $\left(\sum B_{i}\right)^{\omega}$ is a lower face of $\left(\sum A_{i}\right)^{\omega}$ is a mixed subdivision of $\sum A_{i}$ called the coherent mixed subdivision of $\sum A_{i}$ with respect to $\omega$. We denote it by $\mathscr{S}_{\sum}^{\omega} A_{i}$.

In the case where $\sum A_{i}$ is basic, we have the following characterization of $\mathscr{S}_{\sum}^{\omega} A_{i}$. 
Theorem 3.3 (Develin and Sturmfels [3]) Let $\sum A_{i}$ be basic, and let $\omega_{i}: A_{i} \rightarrow \mathbb{R}$ be functions. For any $x=\left(x_{1}, \ldots, x_{m}\right) \in \mathbb{R}^{m}$ and $A_{i}$, let type $\left(x, \omega, A_{i}\right)$ be the set of all $e_{j} \in A_{i}$ such that

$$
x_{j}-\omega_{i}\left(e_{j}\right)=\max \left\{x_{k}-\omega_{i}\left(e_{k}\right): e_{k} \in A_{i}\right\} .
$$

Then $\sum B_{i}$ is an element of $\mathscr{S}_{\sum}^{\omega} A_{i}$ if and only if there is some $x \in \mathbb{R}^{m}$ such that for all $i$, either $B_{i}=\emptyset$ or $B_{i}=\operatorname{type}\left(x, \omega, A_{i}\right)$.

\subsection{The Cayley Trick}

Let $A_{1}, \ldots, A_{n}$ be as before. Let $\Delta^{n-1}=\left\{f_{1}, \ldots, f_{n}\right\}$ be the standard basis of $\mathbb{R}^{n}$. We define the Cayley embedding of $\sum A_{i}$ to be the following set in $\mathbb{R}^{m} \times \mathbb{R}^{n}$ :

$$
\mathcal{C}\left(\sum A_{i}\right):=\bigcup_{i=1}^{n}\left\{\left(x, f_{i}\right): x \in A_{i}\right\}
$$

The Cayley trick says the following.

Theorem 3.4 (Sturmfels [16], Huber et al. [4]) The following are true.

1. $\mathcal{C}$ is a bijection between the mixed cells of $\sum A_{i}$ and the cells of $\mathcal{C}\left(\sum A_{i}\right)$, and this map preserves facial relations.

2. For any mixed subdivision $\mathscr{S}$ of $\sum A_{i}$, the collection $\mathcal{C}(\mathscr{S})$ is a subdivision of $\mathcal{C}\left(\sum A_{i}\right)$. The map $\mathscr{S} \mapsto \mathcal{C}(\mathscr{S})$ is a poset isomorphism between the mixed subdivisions of $\sum A_{i}$ and the subdivisions of $\mathcal{C}\left(\sum A_{i}\right)$.

3. If $\omega_{i}: A_{i} \rightarrow \mathbb{R}$ are functions for $i=1, \ldots, n$ and $\mathcal{C}(\omega): \mathcal{C}\left(\sum A_{i}\right) \rightarrow \mathbb{R}$ is defined as $\mathcal{C}(\omega)\left(x, f_{i}\right)=\omega_{i}(x)$, then

$$
\mathcal{C}\left(\mathscr{S}_{\sum A_{i}}^{\omega}\right)=\mathscr{S}_{\mathcal{C}}^{\mathcal{C}(\omega)}{ }_{\left(\sum A_{i}\right)}
$$

Example 3.5 If $\sum A_{i}$ is basic, then $\mathcal{C}\left(\sum A_{i}\right)$ is a a subset of $\Delta^{m-1} \times \Delta^{n-1}$. If $A_{i}=$ $\Delta^{m-1}$ for all $i$, then $\mathcal{C}\left(\sum A_{i}\right)=\Delta^{m-1} \times \Delta^{n-1}$.

Example 3.6 If $\Pi^{m-1}$ is the $(m-1)$-dimensional permutohedron (see Example 3.2), then $\mathcal{C}\left(\Pi^{m-1}\right)$ can be written as

$$
\mathcal{C}\left(\Pi^{m-1}\right)=\bigcup_{1 \leq i<j \leq m}\left\{\left(e_{i}, f_{i j}\right),\left(e_{j}, f_{i j}\right)\right\} \subset \mathbb{R}^{m} \times \mathbb{R}^{\left(\begin{array}{c}
m \\
2
\end{array}\right)} .
$$

We say that two fine mixed subdivisions $\mathscr{T}$ and $\mathscr{T}^{\prime}$ differ by a fip if the triangulations $\mathcal{C}(\mathscr{T})$ and $\mathcal{C}\left(\mathscr{T}^{\prime}\right)$ differ by a flip. We define the flip graph analogously.

Remark 3.7 Cayley embeddings of zonotopes are Lawrence polytopes. The connectivity of flip graphs on zonotopal tilings of zonotopes can be equivalently stated as the connectivity of flip graphs on triangulations of Lawrence polytopes. 


\section{A Zonotope with Disconnected Flip Graph}

We are now ready to construct a zonotope and a nontrivial component of its flip graph. Explicitly, this zonotope is the three-dimensional permutohedron, but with each of its generating vectors repeated a large number of times. The idea of the proof will be to generate certain random tilings of this zonotope, and then show that most of the generated tilings will be in different components of the flip graph. We begin by developing the machinery needed to generate the random tilings.

\subsection{Zonotopal Tilings of the 3-Permutohedron}

It will be notationally easier to work with Cayley embeddings of zonotopes rather than zonotopes themselves. Thus we will identify a zonotope with its Cayley embedding.

We first set up some notation. For a set $S$, let $\Gamma_{S}^{k}$ denote the set of all ordered $k$-tuples $\left(i_{1}, \ldots, i_{k}\right)$ of distinct $i_{1}, \ldots, i_{k} \in S$ under the equivalence relation $\left(i_{1}, \ldots, i_{k}\right) \sim$ $\left(i_{2}, \ldots, i_{k}, i_{1}\right)$. We will use $\left(i_{1} \cdots i_{k}\right)$ to denote the equivalence class of $\left(i_{1}, \ldots, i_{k}\right)$ in $\Gamma_{S}^{k}$. We write- $\left(i_{1} \cdots i_{k}\right)$ to denote $\left(i_{k} \cdots i_{1}\right)$. We abbreviate $\Gamma_{[n]}^{k}$ as $\Gamma_{n}^{k}$.

The main object we will focus on is the Cayley embedding of the 3-dimensional permutohedron. This object was described in Example 3.6; here we will redefine it using slightly different indices. Let $\left\{e_{1}, \ldots, e_{4}\right\}$ be the standard basis for $\mathbb{R}^{4}$ and let $\left\{f_{\alpha}\right\}_{\alpha \in \Gamma_{4}^{2}}$ be the standard basis for $\mathbb{R}^{6}$. Then

$$
\Pi^{3}:=\bigcup_{(i j) \in \Gamma_{4}^{2}}\left\{\left(e_{i}, f_{(i j)}\right),\left(e_{j}, f_{(i j)}\right)\right\} \subset \mathbb{R}^{4} \times \mathbb{R}^{6}
$$

is the (Cayley embedding of the) 3-dimensional permutohedron. (Note we have written $\Pi^{3}$ instead of $\mathcal{C}\left(\Pi^{3}\right)$ here for convenience.)

Our proof will focus on specific circuits of size 6 in the permutohedron. For any $(i j k) \in \Gamma_{4}^{3}$, we have a circuit $X_{(i j k)}=\left(X_{(i j k)}^{+}, X_{(i j k)}^{-}\right)$in $\Pi^{3}$ with affine dependence relation

$$
\left(e_{i}, f_{(i j)}\right)-\left(e_{j}, f_{(i j)}\right)+\left(e_{j}, f_{(j k)}\right)-\left(e_{k}, f_{(j k)}\right)+\left(e_{k}, f_{(k i)}\right)-\left(e_{i}, f_{(k i)}\right)
$$

and $X_{(i j k)}^{+}, X_{(i j k)}^{-}$defined in terms of this affine relation. Define $\mathscr{T}_{(i j k)}:=\mathscr{T}_{X_{(i j k)}}^{+}$. Note that $X_{-\gamma}=\left(X_{\gamma}^{-}, X_{\gamma}^{+}\right)$and hence $\mathscr{T}_{-\gamma}=\mathscr{T}_{X_{\gamma}}^{-}$.

Remark 4.1 In the zonotope setting, each circuit $X_{(i j k)}$ "corresponds" to a pair of opposite hexagonal facets of the 3-permutohedron, namely the facets generated by $\left\{e_{i}-e_{j}, e_{j}-e_{k}, e_{k}-e_{i}\right\}$; see Fig. 1 . The correspondence is in the following sense: In any tight zonotopal tiling $\mathscr{S}$ of the permutohedron, these facets are tiled in the same way. The Cayley embedding of $\mathscr{S}$ is a triangulation $\mathcal{C}(\mathscr{S})$ of $\Pi^{3}$, and the tiling of the aforementioned facets is determined by the triangulation induced by $\mathcal{C}(\mathscr{S})$ on the circuit $X_{(i j k)}$. 
Fig. 1 Facets (outlined in red) corresponding to the circuit $X_{(i j k)}$

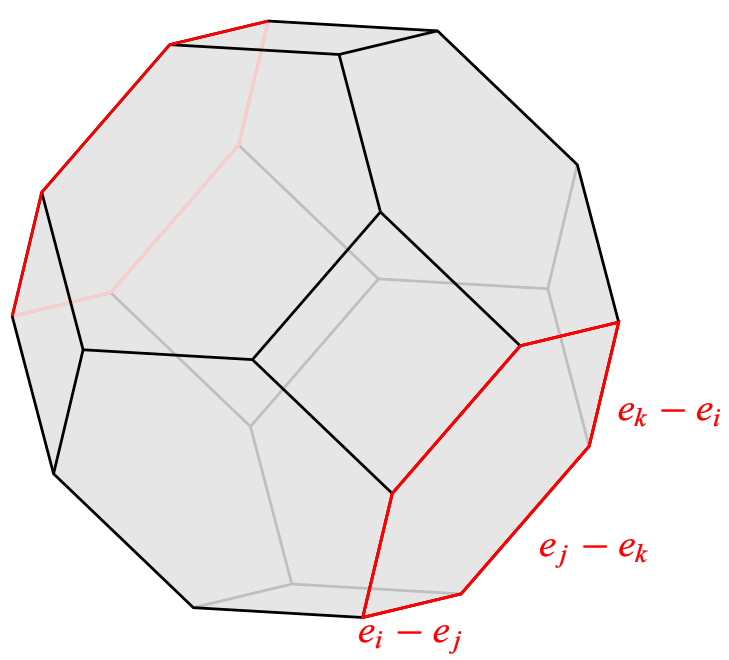

We will now construct eight different tilings of $\Pi^{3}$, each indexed by a different element of $\Gamma_{4}^{3}$. Fix some $\gamma=(i j k) \in \Gamma_{4}^{3}$. Let $\omega: \Pi^{3} \rightarrow \mathbb{R}$ be the function with

$$
\omega\left(e_{i}, f_{(i j)}\right)=\omega\left(e_{j}, f_{(j k)}\right)=\omega\left(e_{k}, f_{(k i)}\right)=1
$$

and $\omega(x)=0$ for all other $x \in \Pi^{3}$. We define $\mathscr{T}_{\Pi^{3}}^{\gamma}:=\mathscr{S}_{\Pi^{3}}^{\omega}$. It is easy to check (using Theorem 3.3, for example) that $\mathscr{T}_{\Pi^{3}}^{\gamma}$ is a triangulation.

Let us take a closer look at $\mathscr{T}_{\Pi^{3}}^{\gamma}$ where $\gamma=(i j k)$. For each $\gamma^{\prime} \in \Gamma_{4}^{3}$, the circuit $X_{\gamma^{\prime}}$ is a face of $\Pi^{3}$, and thus $\mathscr{T}_{\Pi^{3}}^{\gamma}$ contains the triangulation it induces on $X_{\gamma^{\prime}}$. From the definition of $\omega$, we have

$$
\begin{aligned}
\omega\left(e_{i}, f_{(i j)}\right)-\omega\left(e_{j}, f_{(i j)}\right) & +\omega\left(e_{j}, f_{(j k)}\right)-\omega\left(e_{k}, f_{(j k)}\right) \\
& +\omega\left(e_{k}, f_{(k i)}\right)-\omega\left(e_{i}, f_{(k i)}\right)>0
\end{aligned}
$$

and if $l$ is the element of [4] $\backslash\{i, j, k\}$, we have

$$
\begin{aligned}
\omega\left(e_{i}, f_{(i j)}\right)-\omega\left(e_{j}, f_{(i j)}\right) & +\omega\left(e_{j}, f_{(j l)}\right)-\omega\left(e_{l}, f_{(j l)}\right) \\
& +\omega\left(e_{l}, f_{(l i)}\right)-\omega\left(e_{i}, f_{(l i)}\right)>0, \\
\omega\left(e_{j}, f_{(j k)}\right)-\omega\left(e_{k}, f_{(j k)}\right) & +\omega\left(e_{k}, f_{(k l)}\right)-\omega\left(e_{l}, f_{(k l)}\right) \\
& +\omega\left(e_{l}, f_{(l j)}\right)-\omega\left(e_{j}, f_{(l j)}\right)>0, \\
\omega\left(e_{k}, f_{(k i)}\right)-\omega\left(e_{i}, f_{(k i)}\right) & +\omega\left(e_{i}, f_{(i l)}\right)-\omega\left(e_{l}, f_{(i l)}\right) \\
& +\omega\left(e_{l}, f_{(l k)}\right)-\omega\left(e_{k}, f_{(l k)}\right)>0 .
\end{aligned}
$$


Thus, by Proposition $2.4, \mathscr{T}_{\Pi^{3}}^{\gamma}$ induces the following triangulations on the circuits $X_{\gamma^{\prime}}$ :

$$
\mathscr{T}_{(i j k)}, \mathscr{T}_{(i j l)}, \mathscr{T}_{(j k l)}, \mathscr{T}_{(k i l)} \subseteq \mathscr{T}_{\Pi^{3}}^{\gamma}
$$

\subsection{A Group Action on $\Gamma_{4}^{3}$}

The key property of $\mathscr{T}_{\Pi^{3}}^{\gamma}$ is that it only has flips on the circuits $X_{(i j l)}, X_{(j k l)}$, and $X_{(k i l)}$. The idea will be to tile a larger zonotope with 3-permutohedra and then tile each 3-permutohedron with some $\mathscr{T}_{\Pi^{3}}^{\gamma}$ so that in the end, no circuit of size six can be flipped. To help with this construction, we will take some time to define a group action on $\Gamma_{4}^{3}$.

For each $\gamma=(i j k) \in \Gamma_{4}^{3}$, we define a function $o_{\gamma}:\left(\begin{array}{c}{[4]} \\ 3\end{array}\right) \rightarrow \Gamma_{4}^{3}$ by

$$
\begin{gathered}
o_{\gamma}(\{i, j, k\})=(i j k), \\
o_{\gamma}(\{i, j, l\})=(i j l), \\
o_{\gamma}(\{j, k, l\})=(j k l), \\
o_{\gamma}(\{k, i, l\})=(k i l),
\end{gathered}
$$

where $\{l\}=[4] \backslash\{i, j, k\}$. The function $o_{\gamma}$ is a way to "orient" each element of $\left(\begin{array}{c}{[4]} \\ 3\end{array}\right)$ with respect to $\gamma$. Recalling Eq. (4.1), $o_{\gamma}$ is defined in such a way that $\mathscr{T}_{\gamma}(S) \subseteq \mathscr{T}_{\Pi^{3}}$ for all $S \in\left(\begin{array}{c}{[4]} \\ 3\end{array}\right)$. It is easy to check that $\gamma$ is determined by $o_{\gamma}$.

Now, we will map each $\alpha \in \Gamma_{4}^{2}$ to a permutation $\pi_{\alpha}: \Gamma_{4}^{3} \rightarrow \Gamma_{4}^{3}$. This map is completely determined by the following rules: For any distinct $i, j, k, l \in[4]$, we have

$$
\begin{aligned}
& \pi_{(i j)}(i j k)=(j i l), \\
& \pi_{(k l)}(i j k)=(i j l) .
\end{aligned}
$$

We can check that for all distinct $i, j, k, l \in[4]$ and $\gamma \in \Gamma_{4}^{3}$, we have

$$
\begin{gathered}
o_{\pi_{(i j)} \gamma}(\{i, j, k\})=-o_{\gamma}(\{i, j, k\}), \\
o_{\pi_{(i j)} \gamma}(\{i, j, l\})=-o_{\gamma}(\{i, j, l\}), \\
o_{\pi_{(i j)} \gamma}(\{j, k, l\})=o_{\gamma}(\{j, k, l\}), \\
o_{\pi_{(i j)} \gamma}(\{k, i, l\})=o_{\gamma}(\{k, i, l\}) .
\end{gathered}
$$

In other words, $\pi_{(i j)}$ reverses the value of $o_{\gamma}$ on $S \in\left(\begin{array}{c}{[4]} \\ 3\end{array}\right)$ if $\{i, j\} \subseteq S$ and leaves it the same otherwise. Let $G_{\Gamma_{4}^{3}}$ be the permutation group of $\Gamma_{4}^{3}$ generated by all the $\pi_{\alpha}$.

Proposition 4.2 The following are true.

1. Every element of $G_{\Gamma_{4}^{3}}$ is an involution, and $G_{\Gamma_{4}^{3}}$ is abelian and transitive on $\Gamma_{4}^{3}$. 
2. For $l \in[4]$, let $H_{l}$ be the subgroup of $G_{\Gamma_{4}^{3}}$ generated by $\pi_{(i l)}$ for all $i \in[4] \backslash\{l\}$. Let $i, j, k \in[4] \backslash\{l\}$ be distinct, and let $\Gamma_{4}^{3}(i j k)$ be the set of all $\gamma \in \Gamma_{4}^{3}$ such that $o_{\gamma}(\{i, j, k\})=(i j k)$. Then $\Gamma_{4}^{3}(i j k)$ is an orbit of $H_{l}$.

Proof Since each $\gamma$ is determined by $o_{\gamma}$, we can view $G_{\Gamma_{4}^{3}}$ as an action on the set of functions $o_{\gamma}$. It is then clear from (4.2) that we can embed $G_{\Gamma_{4}^{3}}$ as a subgroup of $\mathbb{Z}_{2}^{4}$. This implies that every element of $G_{\Gamma_{4}^{3}}$ is an involution and $G_{\Gamma_{4}^{3}}$ is abelian. It is also easy to check from (4.2) that every element of $\Gamma_{4}^{3}$ has orbit of size 8, and hence $G_{\Gamma_{4}^{3}}$ is transitive.

From (4.2), we see that $H_{l}$ maps $\Gamma_{4}^{3}(i j k)$ to itself and every element of $\Gamma_{4}^{3}(i j k)$ has orbit of size 4 under $H_{l}$. Since $\left|\Gamma_{4}^{3}(i j k)\right|=4, \Gamma_{4}^{3}(i j k)$ is an orbit of $H_{l}$.

\subsection{A Zonotope and a Component of Its Flip Graph}

We are now ready to go into the main proof. Let $N$ be a positive integer to be determined later. For each distinct $i, j \in[4]$ and $-N \leq r \leq N$, we create a variable $f_{i j}^{r}$, and we make the identification of variables

$$
f_{i j}^{r}=f_{j i}^{-r} .
$$

We think of the $f_{i j}^{r}$ as copies of the variable $f_{(i j)}$ defined in Sect. 4.1. Let $\left\{f_{i j}^{r}\right\}_{1 \leq i<j \leq 4,-N \leq r \leq N}$ be the standard basis for $\mathbb{R}^{6(2 N+1)}$. Let

$$
\Pi:=\bigcup_{1 \leq i<j \leq 4} \bigcup_{-N \leq r \leq N}\left\{\left(e_{i}, f_{i j}^{r}\right),\left(e_{j}, f_{i j}^{r}\right)\right\} \subset \mathbb{R}^{4} \times \mathbb{R}^{6(2 N+1)}
$$

be the 3-dimensional permutohedron with $2 N+1$ copies of each generating vector. As a zonotope, $\Pi$ is the unit 3-permutohedron scaled by $2 N+1$. We now prove our first main result.

Theorem 4.3 For large enough $N$, the flip graph of $\Pi$ is not connected.

We will use the following lemma which identifies a component of the flip graph of $\Pi$ based on the triangulations of certain circuits of $\Pi$. For distinct $i, j, k \in[4]$ and for any $-N \leq r, s, t \leq N$, let $X_{i j k}^{r s t}=\left(\left(X_{i j k}^{r s t}\right)^{+},\left(X_{i j k}^{r s t}\right)^{-}\right)$be the circuit with affine dependence relation

$$
\left(e_{i}, f_{i j}^{r}\right)-\left(e_{j}, f_{i j}^{r}\right)+\left(e_{j}, f_{j k}^{s}\right)-\left(e_{k}, f_{j k}^{s}\right)+\left(e_{k}, f_{k i}^{t}\right)-\left(e_{i}, f_{k i}^{t}\right)
$$

and $\left(X_{i j k}^{r s t}\right)^{+},\left(X_{i j k}^{r s t}\right)^{-}$defined in terms of this relation. Let $\mathscr{T}_{i j k}^{r s t}:=\mathscr{T}_{X_{i j k}^{r s t}}^{+}$.

Lemma 4.4 Let $\mathscr{C}$ be a collection of triangulations of the form $\mathscr{T}_{i j k}^{r s t}$ such that for all $\mathscr{T}_{i j k}^{r s t} \in \mathscr{C}$ and $\{l\}=[4] \backslash\{i, j, k\}$, there exist $1 \leq u, v, w \leq N$ such that

$$
\mathscr{T}_{i j l}^{r v(-u)}, \mathscr{T}_{j k l}^{s w(-v)}, \mathscr{T}_{k i l}^{t u(-w)} \in \mathscr{C}
$$


Let $\mathcal{S}_{\mathscr{C}}$ be the set of all triangulations of $\Pi$ which contain every element of $\mathscr{C}$ as a subset. Then $\mathcal{S}_{\mathscr{C}}$ is closed under flips.

The proof of this lemma follows immediately from the following two facts.

Proposition 4.5 Let $\mathscr{T}$ be a triangulation of $\Pi$ such that $\mathscr{T}_{i j k}^{r s t} \subseteq \mathscr{T}$. Let $\mathscr{T}^{\prime}$ be the result of a flip on $\mathscr{T}$ which is not supported on $X_{i j k}^{r s t}$. Then $\mathscr{T}_{i j k}^{r s t} \subseteq \mathscr{T}^{\prime}$.

Proof Suppose the flip from $\mathscr{T}$ to $\mathscr{T}^{\prime}$ is supported on $X=\left(X^{+}, X^{-}\right)$. By Proposition 2.2, if $\sigma \in \mathscr{T}$ and $\sigma \notin \mathscr{T}^{\prime}$, then $\sigma \supseteq X^{-}$. Thus, if $\mathscr{T}_{i j k}^{r s t} \nsubseteq \mathscr{T}^{\prime}$, then we must have $X^{-} \subseteq X_{i j k}^{r s t}$.

On the other hand, the only circuits of $\Pi$ are of the form $X_{i^{\prime} j^{\prime} k^{\prime}}^{r^{\prime} s^{\prime}}$ or

$$
\left(\left\{\left(e_{i^{\prime}}, f_{i^{\prime} j^{\prime}}^{r^{\prime}}\right),\left(e_{j^{\prime}}, f_{i^{\prime} j^{\prime}}^{s^{\prime}}\right)\right\},\left\{\left(e_{j^{\prime}}, f_{i^{\prime} j^{\prime}}^{r^{\prime}}\right),\left(e_{i^{\prime}}, f_{i^{\prime} j^{\prime}}^{s^{\prime}}\right)\right\}\right)
$$

for some $i^{\prime}, j^{\prime}, k^{\prime}, r^{\prime}, s^{\prime}, t^{\prime}$. Of these circuits, the only circuit whose negative part is contained in $X_{i j k}^{r s t}$ is $X_{i j k}^{r s t}$ itself. Since $X \neq X_{i j k}^{r s t}$ by assumption, we must therefore have $X^{-} \nsubseteq X_{i j k}^{r s t}$, and hence $\mathscr{T}_{i j k}^{r s t} \subseteq \mathscr{T}^{\prime}$.

Proposition 4.6 Let $\mathscr{T}$ be a triangulation of $\Pi$, and suppose that there are distinct $i, j, k, l \in[4]$ and $1 \leq r, s, t, u, v, w \leq N$ such that

$$
\mathscr{T}_{i j l}^{r v(-u)}, \mathscr{T}_{j k l}^{s w(-v)}, \mathscr{T}_{k i l}^{t u(-w)} \subseteq \mathscr{T}
$$

Then $\mathscr{T}$ does not have a flip supported on $X_{i j k}^{r s t}$.

Proof Consider the cell

$$
C:=\left\{\left(e_{i}, f_{i l}^{u}\right),\left(e_{l}, f_{i l}^{u}\right),\left(e_{j}, f_{j l}^{v}\right),\left(e_{l}, f_{j l}^{v}\right),\left(e_{k}, f_{k l}^{w}\right),\left(e_{l}, f_{k l}^{w}\right)\right\}
$$

$C$ is a face of $\Pi$, as seen by the linear functional $\phi$ such that

$$
\begin{aligned}
& \phi\left(e_{a}, 0\right)=0 \text { for all } a \text {, } \\
& \phi\left(0, f_{a b}^{p}\right)= \begin{cases}-1 & \text { if } f_{a b}^{p}=f_{i l}^{u}, f_{j l}^{v}, \text { or } f_{k l}^{w}, \\
0 & \text { otherwise } .\end{cases}
\end{aligned}
$$

Since $C$ is a simplex, we must have $C \in \mathscr{T}$. Thus, there is some maximal simplex $\tau \in \mathscr{T}$ such that $C \subseteq \tau$. Since $\tau$ is maximal, it must contain one element from each of the sets

$$
\left\{\left(e_{i}, f_{i j}^{r}\right),\left(e_{j}, f_{i j}^{r}\right)\right\},\left\{\left(e_{j}, f_{j k}^{s}\right),\left(e_{k}, f_{j k}^{s}\right)\right\},\left\{\left(e_{k}, f_{k i}^{t}\right),\left(e_{i}, f_{k i}^{t}\right)\right\}
$$

Suppose $\left(e_{i}, f_{i j}^{r}\right) \in \tau$. Then since $C \subseteq \tau$, we have

$$
\left(X_{i j l}^{r v(-u)}\right)^{+}=\left\{\left(e_{i}, f_{i j}^{r}\right),\left(e_{j}, f_{j l}^{v}\right),\left(e_{l}, f_{l i}^{-u}\right)\right\} \subseteq \tau .
$$


On the other hand, since $\mathscr{T}_{i j l}^{r v(-u)} \subseteq \mathscr{T}$ by assumption, we have $\left(X_{i j l}^{r v(-u)}\right)^{-} \in \mathscr{T}$. This is a contradiction, because the opposite parts of a circuit cannot both be cells of a triangulation (since the interiors of these cells intersect). Hence $\left(e_{j}, f_{i j}^{r}\right) \in \tau$. Similarly, $\left(e_{k}, f_{j k}^{s}\right) \in \tau$ and $\left(e_{i}, f_{k i}^{t}\right) \in \tau$. Hence, $\left(X_{i j k}^{r s t}\right)^{-} \subseteq \tau$.

We thus have $\left(X_{i j k}^{r s t}\right)^{-} \subseteq \tau$ and $\left|X_{i j k}^{r s t} \cap \tau\right|=3<\left|X_{i j k}^{r s t}\right|-2$. By Proposition 2.3, $\mathscr{T}$ does not have a flip supported on $X_{i j k}^{r s t}$.

Theorem 4.3 follows from Lemma 4.4 if there is some $\mathscr{C}$ for which $\mathcal{S}_{\mathscr{C}}$ is neither empty nor the whole set of triangulations of $\Pi$. We show this in the next section.

\subsection{Construction of $\mathscr{C}$ and Some $\mathscr{T} \in \mathcal{S}_{\mathscr{C}}$}

We first consider the regular subdivision $\mathscr{S}_{\Pi}^{\omega}$ where $\omega: \Pi \rightarrow \mathbb{R}$ is a function such that

$$
\omega\left(e_{i}, f_{i j}^{r}\right)-\omega\left(e_{j}, f_{i j}^{r}\right)=r
$$

for all distinct $i, j \in[4]$ and $-N \leq r \leq N$. The cells of $\mathscr{S}_{\Pi}^{\omega}$ are described as follows.

Proposition 4.7 Let $\mathscr{X}$ be the set of $x=\left(x_{1}, x_{2}, x_{3}, x_{4}\right) \in \mathbb{R}^{4}$ such that $x_{1}+\cdots+$ $x_{4}=0$ and if $i j k l$ is a permutation of [4] such that $x_{i} \geq x_{j} \geq x_{k} \geq x_{l}$, then $x_{i}-x_{j}$, $x_{j}-x_{k}$, and $x_{k}-x_{l}$ are integers at most $N$. Let $\mathscr{X}^{*}$ be the set of $x \in \mathscr{X}$ such that $\left|x_{i}-x_{j}\right| \leq N$ for all $i, j \in[n]$. The following are true.

1. The map $C(x)=\left\{\left(e_{i}, f_{i j}^{r}\right): x_{i}-x_{j} \geq r\right\}$ is a bijection from $\mathscr{X}$ to the maximal cells of $\mathscr{S}_{\Pi}^{\omega}$.

2. If $x \in \mathscr{X}^{*}$, then $C(x)$ is the Cayley embedding of a translated 3-permutohedron. Specifically, $C(x)=\Pi(x) \cup D$, where

$$
\Pi(x):=\bigcup_{1 \leq i<j \leq 4}\left\{\left(e_{i}, f_{i j}^{x_{i}-x_{j}}\right),\left(e_{j}, f_{i j}^{x_{i}-x_{j}}\right)\right\}
$$

and $D$ is a simplex affinely independent to $\Pi(x)$.

3. Let $x \in \mathscr{X} \backslash \mathscr{X}^{*}$. Suppose $F_{1}, \ldots, F_{k}$ are faces of $C(x)$ and $\mathscr{T}_{1}, \ldots, \mathscr{T}_{k}$ are triangulations of these faces, respectively, which agree on intersections of these faces. Then there is a triangulation of $C(x)$ which contains $\mathscr{T}_{1}, \ldots, \mathscr{T}_{k}$ as subsets.

Proof We first prove Part 1. By Theorem 3.3, a set $C \subseteq \Pi$ is a cell of $\mathscr{S}_{\Pi}^{\omega}$ if and only if there is some $x \in \mathbb{R}^{4}$ such that

$$
\begin{aligned}
C & =\left\{\left(e_{i}, f_{i j}^{r}\right): x_{i}-\omega\left(e_{i}, f_{i j}^{r}\right) \geq x_{j}-\omega\left(e_{j}, f_{i j}^{r}\right)\right\} \\
& =\left\{\left(e_{i}, f_{i j}^{r}\right): x_{i}-x_{j} \geq r\right\}=C(x) .
\end{aligned}
$$

Moreover, if $C$ is maximal, then there are pairs $(i, j),\left(i^{\prime}, j^{\prime}\right),\left(i^{\prime \prime}, j^{\prime \prime}\right)$ such that $\left\{e_{i}-\right.$ $\left.e_{j}, e_{i^{\prime}}-e_{j^{\prime}}, e_{i^{\prime \prime}}-e_{j^{\prime \prime}}\right\}$ is linearly independent and

$$
\left\{\left(e_{i}, f_{i j}^{r}\right),\left(e_{j}, f_{i j}^{r}\right),\left(e_{i^{\prime}}, f_{i^{\prime} j^{\prime}}^{r^{\prime}}\right),\left(e_{j^{\prime}}, f_{i^{\prime} j^{\prime}}^{r^{\prime}}\right),\left(e_{i^{\prime \prime}}, f_{i^{\prime \prime} j^{\prime \prime}}^{r^{\prime \prime}}\right),\left(e_{j^{\prime \prime}}, f_{i^{\prime \prime} j^{\prime \prime}}^{r^{\prime \prime}}\right)\right\} \subseteq C
$$


Fig. 2 A portion of the tiling associated to $\omega$

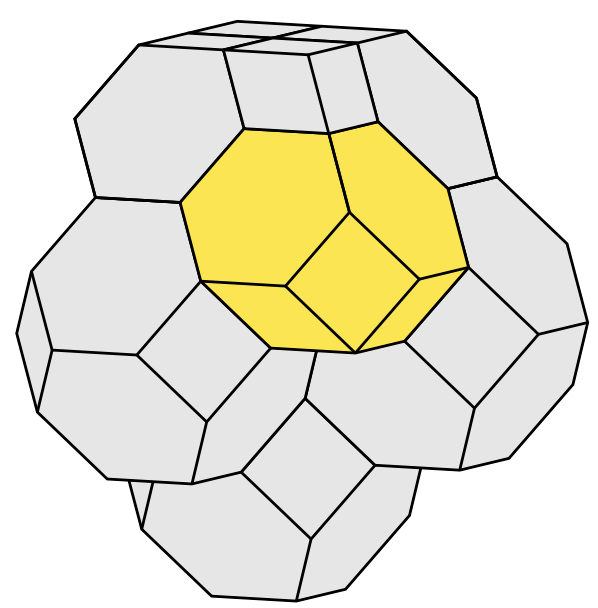

for some $-N \leq r, r^{\prime}, r^{\prime \prime} \leq N$. If $x \in \mathbb{R}^{4}$ is such that $C=C(x)$ for this maximal cell $C$, then (4.4) implies $x_{i}-x_{j}=r, x_{i^{\prime}}-x_{j^{\prime}}=r^{\prime}$, and $x_{i^{\prime \prime}}-x_{j^{\prime \prime}}=r^{\prime \prime}$. By the linear independence of $\left\{e_{i}-e_{j}, e_{i^{\prime}}-e_{j^{\prime}}, e_{i^{\prime \prime}}-e_{j^{\prime \prime}}\right\}$, there is a unique $x$ for which these equalities hold and $x_{1}+\cdots+x_{4}=0$. Moreover, this $x$ is contained in $\mathscr{X}$. Finally, for any $x \in \mathscr{X}, C(x)$ is a maximal cell of $\mathscr{S}_{\Pi}^{\omega}$. This proves Part 1. Part 2 follows immediately.

The only nontrivial case of Part 3 is when $x$ satisfies $x_{i} \geq x_{j} \geq x_{k} \geq x_{l}$ for some permutation $i j k l$ of [4], $x_{i}-x_{k} \leq N, x_{j}-x_{l} \leq N$, and $x_{i}-x_{l}>N$. (A zonotopal tile corresponding to such a cell is highlighted in yellow in Fig. 2.) In this case $C(x)$ is of the form $X_{i j k}^{r s t} \cup X_{j k l}^{s u v} \cup D$, where $D$ is a simplex affinely independent to $X_{i j k}^{r s t} \cup X_{j k l}^{s u v}$. By Proposition 5.9 (or by an easy check), any triangulations of $X_{i j k}^{r s t}$ and $X_{j k l}^{s u v}$ can be extended to a triangulation of $X_{i j k}^{r s t} \cup X_{j k l}^{s u v}$, and hence to a triangulation of $C(x)$.

Remark 4.8 In terms of mixed subdivisions, the coherent mixed subdivision associated to $\omega$ is given by tiling the large permutohedron $\Pi$ with smaller unit permutohedra (the cells corresponding to $\mathscr{X}^{*}$ ) and pieces of permutohedra (the tiles corresponding to $\left.\mathscr{X} \backslash \mathscr{X}^{*}\right)$. Each point $x \in \mathscr{X}$ is the center of the zonotopal tile corresponding to $C(x)$.

We now give a brief overview of the rest of the proof. The goal is to construct a triangulation which refines $\mathscr{S}_{\Pi}^{\omega}$ and which is sufficiently "complicated". The idea is to start with a known triangulation and apply the group action $G_{\Gamma_{4}^{3}}$ to the triangulations of the cells $C(x)$ in a way that produces another triangulation of $\Pi$. As we will show, by randomly doing this process we can produce triangulations from our original triangulation that cannot be produced through sequences of flips.

For each $x \in \mathscr{X}^{*}$, we have an affine isomorphism $\Pi^{3} \rightarrow \Pi(x)$ given by $f_{(i j)} \mapsto$ $f_{i j}^{x_{i}-x_{j}}$. For each $\gamma \in \Gamma_{4}^{3}$, let $\mathscr{T}_{\Pi(x)}^{\gamma}$ be the image of $\mathscr{T}_{\Pi^{3}}^{\gamma}$ under this isomorphism.

We will now choose a random triangulation of every $C(x), x \in \mathscr{X}^{*}$, as follows: 
1. For each $1 \leq i<j \leq 4$ and $-N \leq r \leq N$, let $g_{i j}^{r}=g_{j i}^{-r}$ be an independent random element of $G_{\Gamma_{4}^{3}}$ which is 1 with probability $1 / 2$ and $\pi_{(i j)}$ with probability $1 / 2$.

2. For each $x \in \mathscr{X}^{*}$, triangulate $\Pi(x)$ by $\mathscr{T}_{\Pi(x)}^{\gamma(x)}$, where

$$
\gamma(x):=\left(\prod_{1 \leq i<j \leq 4} g_{i j}^{x_{i}-x_{j}}\right) .
$$

3. Extend $\mathscr{T}_{\Pi(x)}^{\gamma(x)}$ uniquely to a triangulation $\mathscr{T}_{C(x)}$ of $C(x)$.

Proposition 4.9 For any two $x, x^{\prime} \in \mathscr{X}^{*}$, the triangulations $\mathscr{T}_{C(x)}$ and $\mathscr{T}_{C\left(x^{\prime}\right)}$ agree on the common face of $C(x)$ and $C\left(x^{\prime}\right)$.

Proof The only non-trivial case is when $C(x) \cap C\left(x^{\prime}\right)$ contains a circuit $X_{i j k}^{r s t}$. We need to check that $\mathscr{T}_{C(x)}$ and $\mathscr{T}_{C\left(x^{\prime}\right)}$ agree on this circuit. If $X_{i j k}^{r s t} \subseteq C(x) \cap C\left(x^{\prime}\right)$, then

$$
\begin{aligned}
& x_{i}-x_{j}=x_{i}^{\prime}-x_{j}^{\prime}=r, \\
& x_{j}-x_{k}=x_{j}^{\prime}-x_{k}^{\prime}=s, \\
& x_{k}-x_{i}=x_{k}^{\prime}=x_{i}^{\prime}=t .
\end{aligned}
$$

On the other hand, by Proposition 4.2(2), $o_{\gamma(x)}(\{i, j, k\})$ depends only on $g_{i j}^{x_{i}-x_{j}}$, $g_{j k}^{x_{j}-x_{k}}$, and $g_{k i}^{x_{k}-x_{i}}$. It follows that $o_{\gamma(x)}(\{i, j, k\})=o_{\gamma\left(x^{\prime}\right)}(\{i, j, k\})$. Thus $\mathscr{T}_{\Pi(x)}^{\gamma(x)}$ and $\mathscr{T}_{\Pi\left(x^{\prime}\right)}^{\gamma\left(x^{\prime}\right)}$ contain the same triangulation of $X_{i j k}^{r s t}$, as desired.

By Proposition 4.9 and Proposition 4.7 (3), we can thus extend the above triangulations of the $C(x)$ to a full triangulation of $\Pi$. Call this triangulation $\mathscr{T}$.

Let $\mathscr{C}$ be the collection of all triangulations $\mathscr{T}_{i j k}^{r s t} \subseteq \mathscr{T}$ with $i, j, k \in[4]$ distinct, $-N \leq r, s, t \leq N$, and $r+s+t=0$. We prove that $\mathscr{C}$ satisfies the hypotheses of Lemma 4.4. We will actually prove the following stronger statement, which we will need in the next section. (For the current proof, we only need the second sentence of (B).)

Proposition 4.10 For large enough $N$, with probability greater than $0, \mathscr{T}$ and $\mathscr{C}$ satisfy the following:

(A) For every distinct $i, j, k \in[4]$ and $-N \leq r \leq N$, there exist $-N \leq s, t \leq N$ such that $\mathscr{T}_{i j k}^{r s t} \in \mathscr{C}$.

(B) For every $\mathscr{T}_{i j k}^{r s t} \in \mathscr{C}$ and $\gamma \in \Gamma_{4}^{3}(i j k)$, there exists $x \in \mathscr{X}^{*}$ such that $X_{i j k}^{r s t} \subseteq$ $\Pi(x)$ and $\mathscr{T}[\Pi(x)]=\mathscr{T}_{\Pi(x)}^{\gamma}$. In particular, when $\gamma=(i j k)$, this implies there is some $-N \leq u, v, w \leq N$ such that 


$$
\mathscr{T}_{i j l}^{r v(-u)}, \mathscr{T}_{j k l}^{s w(-v)}, \mathscr{T}_{k i l}^{t u(-w)} \in \mathscr{C}
$$

where $\{l\}=[4] \backslash\{i, j, k\}$.

Proof First, note that for any distinct $i, j, k \in[4]$ and $-N \leq r, s, t \leq N$ with $r+s+t=0$, there is some $x \in \mathscr{X}^{*}$ such that $X_{i j k}^{r s t} \subseteq \Pi(x)$. Hence $\mathscr{C}$ contains a triangulation of $X_{i j k}^{r s t}$. Letting $\gamma=g_{i j}^{r} g_{j k}^{s} g_{k i}^{t}(123)$, we have

$$
\mathscr{T}_{i j k}^{r s t} \in \mathscr{C} \text { if and only if } o_{\gamma}(\{i, j, k\})=(i j k) .
$$

We first bound the probability that (A) does not hold. Fix distinct $i, j, k \in[4]$ and $-N \leq r \leq N$. Let $H$ be the set of all ordered pairs $(s, t) \in[-N, N]^{2}$ with $r+s+t=0$. Note that $|H| \geq N$. For each $(s, t) \in H$ and $\gamma(s, t):=g_{i j}^{r} g_{j k}^{s} g_{k i}^{t}(123)$, it is easy to see from the proof of Proposition 4.2 that $o_{\gamma(s, t)}(\{i, j, k\})=(i j k)$ with probability $1 / 2$. In fact, this probability does not change if we fix $g_{i j}^{r}$, so for all $(s, t) \in H$ these probabilities are mutually independent. Now, from (4.5), there does not exist $(s, t) \in H$ such that $\mathscr{T}_{i j k}^{r s t} \in \mathscr{C}$ if and only if $o_{\gamma(s, t)}(\{i, j, k\}) \neq(i j k)$ for all $(s, t) \in H$. The probability this happens is

$$
\left(\frac{1}{2}\right)^{|H|} \leq\left(\frac{1}{2}\right)^{N} .
$$

By the union bound, the probability that this happens for some distinct $i, j, k \in[4]$ and $-N \leq r \leq N$ is at most

$$
24(2 N+1)\left(\frac{1}{2}\right)^{N}
$$

This gives an upper bound on the probability of (A) not happening.

We now do the same for (B). Fix $\mathscr{T}_{i j k}^{r s t} \in \mathscr{C}$ and $\gamma \in \Gamma_{4}^{3}(i j k)$. Let $H$ be the set of all $x \in \mathscr{X}^{*}$ such that $X_{i j k}^{r s t} \subseteq \Pi(x)$. Note that $|H| \geq N$. Let $\{l\}=[4] \backslash\{i, j, k\}$.

We have $\mathscr{T}_{i j k}^{r s t} \in \mathscr{C}$, which happens if and only if $g_{i j}^{r} g_{j k}^{s} g_{k i}^{t}(123) \in \Gamma_{4}^{3}(i j k)$. Suppose we fix $g_{i j}^{r}, g_{j k}^{s}$, and $g_{k i}^{t}$ such that $g_{i j}^{r} g_{j k}^{s} g_{k i}^{t}(123) \in \Gamma_{4}^{3}(i j k)$. Then for each $x \in H$, it follows from Proposition 4.2 and the definition of $\gamma(x)$ that $\gamma(x)=\gamma$ with probability $1 / 4$. Moreover, since $\left(x_{i}-x_{l}, x_{j}-x_{l}, x_{k}-x_{l}\right)$ is different for each $x \in H$, these probabilities are mutually independent for all $x \in H$. Now, for each $x \in \mathscr{X}^{*}$ we have $\mathscr{T}[\Pi(x)]=\mathscr{T}_{\Pi(x)}^{\gamma(x)}$. Thus the probability that there is no $x \in H$ with $\mathscr{T}[\Pi(x)]=\mathscr{T}_{\Pi(x)}^{\gamma}$ is

$$
\left(\frac{3}{4}\right)^{|H|} \leq\left(\frac{3}{4}\right)^{N}
$$

The probability that this occurs for some distinct $i, j, k \in[4],-N \leq r, s, t \leq N$ with $r+s+t=0$, and $\gamma \in \Gamma_{4}^{3}(i j k)$ is thus at most

$$
96(2 N+1)^{2}\left(\frac{3}{4}\right)^{N}
$$


Hence, the probability that either (A) or (B) does not hold is at most

$$
24(2 N+1)\left(\frac{1}{2}\right)^{N}+96(2 N+1)^{2}\left(\frac{3}{4}\right)^{N}
$$

which for large enough $N$ (specifically, $N \geq 48$ ) is less than 1 .

Thus there exists $\mathscr{C}$ which satisfies the hypotheses of Lemma 4.4 and $\mathscr{T} \in \mathcal{S}_{\mathscr{C}}$. There are triangulations of $\Pi$ which are not in $\mathcal{S}_{\mathscr{C}}$; for example, a different choice of the $g_{i j}^{r}$ would yield a triangulation which does not contain every element of $\mathscr{C}$. This proves Theorem 4.3.

\section{A Product of Two Simplices with Disconnected Flip Graph}

We will use the construction from the previous section to show that the product of two simplices does not in general have connected flip graph. The idea will be to go up one dimension and construct multiple copies of $\mathscr{T}$ in different directions in this space.

\subsection{A Component of the Flip Graph}

For each $\alpha \in \Gamma_{5}^{2}$, construct a finite set $\Delta_{\alpha}$ of variables. We will determine the size of this set later. Let $\Delta^{4}:=\left\{e_{1}, \ldots, e_{5}\right\}$ be the standard basis for $\mathbb{R}^{5}$, and let $\Delta^{n-1}:=\bigcup_{\alpha \in \Gamma_{5}^{2}} \Delta_{\alpha}$ be the standard basis for $\mathbb{R}^{n}$, where $n=\sum_{\alpha \in \Gamma_{5}^{2}}\left|\Delta_{\alpha}\right|$. Let $A:=\Delta^{4} \times \Delta^{n-1}$. We prove the following.

Theorem 5.1 For large enough n, the flip graph of $A$ is not connected.

We first identify a component of the flip graph of $A$. For distinct $i_{1}, \ldots, i_{t} \in[5]$ and distinct $f_{1}, \ldots, f_{t} \in \Delta^{n-1}$, let

$$
X_{i_{1} \cdots i_{t}}^{f_{1} \cdots f_{t}}=\left(\left(X_{i_{1} \cdots i_{t}}^{f_{1} \cdots f_{t}}\right)^{+},\left(X_{i_{1} \cdots i_{t}}^{f_{1} \cdots f_{t}}\right)^{-}\right)
$$

be the circuit in $A$ with affine dependence relation

$$
\left(e_{i_{1}}, f_{1}\right)-\left(e_{i_{2}}, f_{1}\right)+\left(e_{i_{2}}, f_{2}\right)-\left(e_{i_{3}}, f_{3}\right)+\cdots+\left(e_{i_{t}}, f_{t}\right)-\left(e_{i_{1}}, f_{t}\right)
$$

and $\left(X_{i_{1} \cdots i_{t}}^{f_{1} \cdots f_{t}}\right)^{+},\left(X_{i_{1} \cdots i_{t}}^{f_{1} \cdots f_{t}}\right)^{-}$defined in terms of this relation. Let

$$
\mathscr{T}_{i_{1} \cdots i_{t}}^{f_{1} \cdots f_{t}}:=\mathscr{T}_{X_{i_{1} \cdots i_{t}}^{f_{1} \cdots f_{t}}}^{+} .
$$

If $f_{1} \in \Delta_{\left(i_{1} i_{2}\right)}, f_{2} \in \Delta_{\left(i_{2} i_{3}\right)}, \ldots, f_{t} \in \Delta_{\left(i_{t} i_{1}\right)}$, then we call the circuit $X_{i_{1} \cdots i_{t}}^{f_{1} \cdots f_{t}}$ and the triangulation $\mathscr{T}_{i_{1} \cdots i_{t}}^{f_{1} \cdots f_{t}}$ zonotopal. We now identify a component of the flip graph of $A$. 
Lemma 5.2 For each $S \in\left(\begin{array}{c}{[5]} \\ 4\end{array}\right)$ and distinct $i, j \in S$, let $\mathscr{C}_{S, i, j}$ be a collection of zonotopal triangulations of the form $\mathscr{T}_{i_{1} i_{2} i_{3}}^{f_{1} f_{2} f_{3}}$ where $i_{1}, i_{2}, i_{3} \in S$. Assume that for each $S \in\left(\begin{array}{c}{[5]} \\ 4\end{array}\right)$ and distinct $i, j \in S$, and for each $\mathscr{T}_{i_{1} i_{2} i_{3}}^{f_{1} f_{2} f_{3}} \in \mathscr{C}_{S, i, j}$, the following are true.

1. If $\left\{i_{4}\right\}=S \backslash\left\{i_{1}, i_{2}, i_{3}\right\}$, then there exist $f_{1}^{\prime} \in \Delta_{\left(i_{1} i_{4}\right)}, f_{2}^{\prime} \in \Delta_{\left(i_{2} i_{4}\right)}$, and $f_{3}^{\prime} \in \Delta_{\left(i_{3} i_{4}\right)}$ such that

$$
\mathscr{T}_{i_{1} i_{2} i_{4}}^{f_{1} f_{2}^{\prime} f_{1}^{\prime}}, \mathscr{T}_{i_{2} i_{3} i_{4}}^{f_{2} f_{3}^{\prime} f_{2}^{\prime}}, \mathscr{T}_{i_{3} i_{1} i_{4}}^{f_{3} f_{1}^{\prime} f_{3}^{\prime}} \in \mathscr{C}_{S, i, j}
$$

2. If $i_{1}=i, i_{2}=j$, and $i_{4}$ is as above, then there exist $f_{1}^{\prime} \in \Delta_{\left(i_{1} i_{4}\right)}, f_{2}^{\prime} \in \Delta_{\left(i_{2} i_{4}\right)}$, and $f_{3}^{\prime} \in \Delta_{\left(i_{3} i_{4}\right)}$ such that

$$
\mathscr{T}_{i_{1} i_{2} i_{4}}^{f_{1} f_{2}^{\prime} f_{1}^{\prime}}, \mathscr{T}_{i_{3} i_{2} i_{4}}^{f_{2} f_{2}^{\prime} f_{3}^{\prime}}, \mathscr{T}_{i_{1} i_{3} i_{4}}^{f_{3} f_{3}^{\prime} f_{1}^{\prime}} \in \mathscr{C}_{S, i, j}
$$

In addition, assume the following about the $\mathscr{C}_{S, i, j}$.

3. For each distinct $i, j, l \in[5]$ and $f_{1} \in \Delta_{(i j)}$, there exist $k \in[5] \backslash\{i, j, l\}, f_{2} \in$ $\Delta_{(j k)}$, and $f_{3} \in \Delta_{(k i)}$ such that

$$
\mathscr{T}_{i j k}^{f_{1} f_{2} f_{3}} \in \mathscr{C}_{[5] \backslash\{l\}, i, j}
$$

Let $\mathcal{S}_{\mathscr{C}}$ be the set of all triangulations $\mathscr{T}$ of A satisfying the following.

(i) For each $S \in\left(\begin{array}{c}{[5]} \\ 4\end{array}\right)$, distinct $i, j \in S$, and $\mathscr{T}_{i_{1} i_{2} i_{3}}^{f_{1} f_{3} f_{3}} \in \mathscr{C}_{S, i, j}$, we have

$$
\mathscr{T}_{i_{1} i_{2} i_{3}}^{f_{1} f_{2} f_{3}} \subseteq \mathscr{T}
$$

(ii) For each $S \in\left(\begin{array}{c}{[5]} \\ 4\end{array}\right)$, distinct $i, j \in S$, and $\mathscr{T}_{i j k}^{f_{1} f_{2} f_{3}} \in \mathscr{C}_{S, i, j}$ where $k \in S \backslash\{i, j\}$, if $\{l\}=[5] \backslash S$, then for any $f \in \Delta_{(i l)}$ we have

$$
X_{i j k}^{f_{1} f_{2} f_{3}} \backslash\left\{\left(e_{i}, f_{1}\right)\right\} \cup\left\{\left(e_{i}, f\right)\right\} \in \mathscr{T} .
$$

Then $\mathcal{S}_{\mathscr{C}}$ is closed under flips.

Proof We need the following two facts about triangulations of $A$. They are analogous to Propositions 4.5 and 4.6. We defer their proofs to the Appendix.

Proposition 5.3 Let $\mathscr{T}$ be a triangulation of $A$ and let $\mathscr{T}^{\prime}$ be the result of a flip on $\mathscr{T}$ supported on $X=\left(X^{+}, X^{-}\right)$. Suppose that $\sigma \in \mathscr{T}$ and $\sigma \notin \mathscr{T}^{\prime}$, and $G(\sigma)$ is connected. Then $\sigma$ contains a maximal simplex of $\mathscr{T}_{X}^{+}$.

Proposition 5.4 Let $\mathscr{T}$ be a triangulation of A. Let $i, j, k, l \in[5]$ be distinct, and for each $\alpha \in \Gamma_{\{i, j, k, l\}}^{2}$, let $f_{\alpha} \in \Delta^{n-1}$. Suppose that

$$
\mathscr{T}_{j k l}^{f_{(j k)} f_{(k l)} f_{(l j)}}, \mathscr{T}_{k i l}^{f_{(k i)} f_{(i l)} f_{(l k)}} \subseteq \mathscr{T}
$$


and

$$
X_{i j l}^{f_{(i j)} f_{(j l)} f_{(l i)}} \backslash\left\{\left(e_{i}, f_{(i j)}\right)\right\} \in \mathscr{T} .
$$

Then $\mathscr{T}$ does not have a flip supported on $X_{i j k}^{f_{(i j)} f_{(j k)} f_{(k i)}}$.

We also need the following two facts about flips on elements of $\mathcal{S}_{\mathscr{C}}$.

Proposition 5.5 Let $i, j, l \in[5]$ be distinct and let $f_{1} \in \Delta_{(i j)}, f_{2} \in \Delta_{(i l)}$. Let $\mathscr{T} \in \mathcal{S}_{\mathscr{C}}$. Then $\mathscr{T}$ does not have a flip supported on $X_{i j}^{f_{1} f_{2}}$.

Proof By Property 3 of $\mathscr{C}_{S, i, j}$, there exist $k \in[5] \backslash\{i, j, l\}, f_{2}^{\prime} \in \Delta_{(j k)}$, and $f_{3}^{\prime} \in \Delta_{(k i)}$ such that $\mathscr{T}_{i j k}^{f_{1} f_{2}^{\prime} f_{3}^{\prime}} \in \mathscr{C}_{[5] \backslash\{l\}, i, j}$. By property (ii) of $\mathcal{S}_{\mathscr{C}}$, it follows that

$$
\sigma:=X_{i j k}^{f_{1} f_{2}^{\prime} f_{3}^{\prime}} \backslash\left\{\left(e_{i}, f_{1}\right)\right\} \cup\left\{\left(e_{i}, f_{2}\right)\right\} \in \mathscr{T} .
$$

If $\tau$ is a maximal simplex in $\mathscr{T}$ containing $\sigma$, then $\left(X_{i j}^{f_{1} f_{2}}\right)^{-} \subseteq \tau$ but $\left|X_{i j}^{f_{1} f_{2}} \cap \tau\right|=2$. So by Proposition $2.3, \mathscr{T}$ does not have a flip supported on $X_{i j}^{f_{1} f_{2}}$.

Proposition 5.6 Let $\mathscr{T}_{i_{1} i_{2} i_{3}}^{f_{1} f_{2} f_{3}} \in \mathscr{C}_{S, i, j}$. Let $\mathscr{T} \in \mathcal{S}_{\mathscr{C}}$. Then $\mathscr{T}$ does not have a flip supported on $X_{i_{1} i_{2} i_{3}}^{f_{1} f_{3} f_{3}}$.

Proof This is a direct corollary of Property 1 of $\mathscr{C}_{S, i, j}$ and Proposition 5.4.

We now proceed with the proof. Suppose that $\mathscr{T} \in \mathcal{S}_{\mathscr{C}}$, and let $\mathscr{T}^{\prime}$ be the result of a flip on $\mathscr{T}$ supported on $X=\left(X^{+}, X^{-}\right)$. We prove that properties (i) and (ii) hold for $\mathscr{T}^{\prime}$.

Property (i). Suppose that $\mathscr{T}_{i_{1} i_{2} i_{3}}^{f_{1} f_{2} f_{3}} \in \mathscr{C}_{S, i, j}$ and $\mathscr{T}_{i_{1} i_{2} i_{3}}^{f_{1} f_{2} f_{3}} \nsubseteq \mathscr{T}^{\prime}$. Without loss of generality, assume that $X_{i_{1} i_{2} i_{3}}^{f_{1} f_{2} f_{3}} \backslash\left\{\left(e_{i_{1}}, f_{1}\right)\right\} \notin \mathscr{T}^{\prime}$. By Proposition 5.3, $X_{i_{1} i_{2} i_{3}}^{f_{1} f_{2} f_{3}} \backslash\left\{\left(e_{i_{1}}, f_{1}\right)\right\}$ contains a maximal simplex of $\mathscr{T}_{X}^{+}$. This leaves two cases for $X$.

Case 1: $X$ has size 4 . Thus we can write $X=X_{i_{1}^{\prime} i_{2}^{\prime}}^{f^{\prime} f_{2}^{\prime}}$ for some $i_{1}^{\prime}, i_{2}^{\prime} \in\left\{i_{1}, i_{2}, i_{3}\right\}$, $f_{1}^{\prime} \in \Delta_{\left(i_{1}^{\prime} i_{2}^{\prime}\right)}$, and $f_{2}^{\prime} \in \Delta_{\left(i_{1}^{\prime} i_{3}^{\prime}\right)}$, where $\left\{i_{3}^{\prime}\right\}=\left\{i_{1}, i_{2}, i_{3}\right\} \backslash\left\{i_{1}^{\prime}, i_{2}^{\prime}\right\}$. However, this contradicts Proposition 5.5. So we cannot have $|X|=4$.

Case 2: $X=X_{i_{1} i_{2} i_{3}}^{f_{1} f_{2} f_{3}}$. This contradicts Proposition 5.6.

Hence we must have $\mathscr{T}_{i_{1} i_{2} i_{3}}^{f_{1} f_{2} f_{3}} \subseteq \mathscr{T}^{\prime}$, as desired.

Property (ii). Suppose that $\sigma:=X_{i j k}^{f_{1} f_{2} f_{3}} \backslash\left\{\left(e_{i}, f_{1}\right)\right\} \cup\left\{\left(e_{i}, f\right)\right\} \notin \mathscr{T}^{\prime}$, with variables as defined in (ii). By Proposition 5.3, $\sigma$ contains a maximal simplex of $X$. By the same argument as in part (i), we cannot have $|X|=4$ or $X=X_{i j k}^{f_{1} f_{2} f_{3}}$. This leaves

$$
X=X_{j i k}^{f f_{3} f_{2}}
$$

as the only possibility. 
We show that $\mathscr{T}$ cannot have a flip on this circuit. Let $l^{\prime}=S \backslash\{i, j, k\}$. By Property 2 of $\mathscr{C}_{S, i, j}$, there exist $f_{1}^{\prime} \in \Delta_{\left(i l^{\prime}\right)}, f_{2}^{\prime} \in \Delta_{\left(j l^{\prime}\right)}, f_{3}^{\prime} \in \Delta_{\left(k l^{\prime}\right)}$ such that

$$
\mathscr{T}_{i j l^{\prime}}^{f_{1} f_{2}^{\prime} f_{1}^{\prime}}, \mathscr{T}_{k j l^{\prime}}^{f_{2} f_{2}^{\prime} f_{3}^{\prime}}, \mathscr{T}_{i k l^{\prime}}^{f_{3} f_{3}^{\prime} f_{1}^{\prime}} \in \mathscr{C}_{S, i, j}
$$

By Property (ii), the first of these inclusions implies that

$$
X_{i j l^{\prime}}^{f_{1} f_{2}^{\prime} f_{1}^{\prime}} \backslash\left\{\left(e_{i}, f_{1}\right)\right\} \cup\left\{\left(e_{i}, f\right)\right\} \in \mathscr{T}
$$

and hence

$$
X_{j i l^{\prime}}^{f f_{2}^{\prime} f_{2}^{\prime}} \backslash\left\{\left(e_{j}, f\right)\right\} \in \mathscr{T} .
$$

This inclusion along with the last two inclusions of (5.1) imply, by Proposition 5.4, that $\mathscr{T}$ does not have a flip on $X_{j i k}^{f f_{3} f_{2}}$, as desired.

\subsection{Reduction to Zonotopes}

Let

$$
\Pi:=\bigcup_{(i j) \in \Gamma_{5}^{2}} \bigcup_{f \in \Delta_{(i j)}}\left\{\left(e_{i}, f\right),\left(e_{j}, f\right)\right\}
$$

be the large 4-permutohedron embedded in $A$. Suppose we have collections $\mathscr{C}_{S, i, j}$ which satisfy the conditions of Lemma 5.2. Let $\mathscr{T}$ be a triangulation of $\Pi$. Notice that if Properties (i) and (ii) of Lemma 5.2 hold for $\mathscr{T}$, then they hold for any collection containing $\mathscr{T}$. In particular, if $\mathscr{T}$ can be extended to a triangulation $\mathscr{T}^{\prime}$ of $A$, then we will have some $\mathscr{T}^{\prime} \in \mathcal{S}_{\mathscr{C}}$. The next proposition guarantees we can always do this.

Proposition 5.7 If $\mathscr{T}$ is a triangulation of $\Pi$, then there is a triangulation $\mathscr{T}^{\prime}$ of $A$ with $\mathscr{T} \subseteq \mathscr{T}^{\prime}$.

Proof Let $\mathscr{S}_{A}:=\mathscr{S}_{A}^{\omega}$ be the regular subdivision of $A$ with $\omega: A \rightarrow \mathbb{R}$ defined as follows. For each distinct $i, j, k \in[5]$ and $f \in \Delta_{(i j)}$, let $\epsilon_{f, k}>0$ be a generic positive real number, and define

$$
\omega\left(e_{i}, f\right)=0, \quad \omega\left(e_{j}, f\right)=0, \quad \omega\left(e_{k}, f\right)=\epsilon_{f, k} .
$$

Then $\mathscr{S}_{A}$ contains $\Pi$ as a cell, corresponding to $x=0$ under the notation of Theorem 3.3. Since the $\epsilon_{f, k}$ are generic, all other cells of $\mathscr{S}_{A}$ can be written as $F \cup D$, where $F$ is a face of $\Pi$ and $D$ is a simplex affinely independent to $F$. Hence, any triangulation of the cell $\Pi$ can be extended to a refinement of $\mathscr{S}_{A}$ which is a triangulation of $A$. 


\subsection{Construction of a Zonotopal Tiling}

We now construct a triangulation of $\Pi$ from which we will obtain our collections $\mathscr{C}_{S, i, j}$. For each $(i j) \in \Gamma_{5}^{2}$, partition $\Delta_{(i j)}$ into the following sets: $\Delta_{i, j}, \Delta_{j, i}$, and

$$
\begin{aligned}
& \Delta_{S, i^{\prime}, j^{\prime},(i j)} \text { for each } S \in\left(\begin{array}{c}
{[5]} \\
4
\end{array}\right) \text { with } i, j, \in S \\
& \text { and distinct } i^{\prime}, j^{\prime} \in S \text { with }\left\{i^{\prime}, j^{\prime}\right\} \neq\{i, j\} .
\end{aligned}
$$

Next, for each distinct $i, j \in[5]$, choose an element $f_{i, j}^{*} \in \Delta_{i, j}$. For each $S \in\left(\begin{array}{c}{[5]} \\ 4\end{array}\right)$ with $i, j \in S$, let $\Delta_{S, i, j,(i j)} \subseteq \Delta_{i, j}$ be sets such that

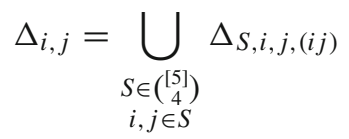

and

$$
\Delta_{S, i, j,(i j)} \cap \Delta_{S^{\prime}, i, j,(i j)}=\left\{f_{i, j}^{*}\right\} \quad \text { for each distinct } S, S^{\prime} \in\left(\begin{array}{c}
{[5]} \\
4
\end{array}\right), \quad i, j \in S, S^{\prime} .
$$

The sizes of all of these sets will be determined later.

Now, we let $\mathscr{S}:=\mathscr{S}_{\Pi}^{\omega}$ be the regular subdivision of $\Pi$ with $\omega: \Pi \rightarrow \mathbb{R}$ defined as follows. First, for every distinct $i, j \in[5]$, we set

$$
\omega\left(e_{i}, f\right)=0, \quad \omega\left(e_{j}, f\right)=1 \quad \text { if } f \in \Delta_{i, j} .
$$

Finally, for each $S \in\left(\begin{array}{c}{[5]} \\ 4\end{array}\right)$ and distinct $i, j, k \in S$, let $0<\epsilon_{S, i, j, k}<1$ be a generic real number. We set

$$
\begin{array}{lll}
\omega\left(e_{i}, f\right)=0, & \omega\left(e_{k}, f\right)=\epsilon_{S, i, j, k} & \text { if } f \in \Delta_{S, i, j,(i k)} \text { and } k \neq j, \\
\omega\left(e_{k}, f\right)=\epsilon_{S, i, j, k}, & \omega\left(e_{j}, f\right)=1 & \text { if } f \in \Delta_{S, i, j,(k j)} \text { and } k \neq i, \\
\omega\left(e_{k}, f\right)=\epsilon_{S, i, j, k}, & \omega\left(e_{k^{\prime}}, f\right)=\epsilon_{S, i, j, k^{\prime}} & \text { if } f \in \Delta_{S, i, j,\left(k k^{\prime}\right)} \text { and } k, k^{\prime} \neq i, j .
\end{array}
$$

We analyze the cells of $\mathscr{S}$. For each $S \in\left(\begin{array}{c}{[5]} \\ 4\end{array}\right)$ and distinct $i, j \in S$, let

$$
\Pi_{S, i, j}:=\bigcup_{\left(i^{\prime} j^{\prime}\right) \in \Gamma_{S}^{2}} \bigcup_{f \in \Delta_{S, i, j,\left(i^{\prime} j^{\prime}\right)}}\left\{\left(e_{i^{\prime}}, f\right),\left(e_{j^{\prime}}, f\right)\right\}
$$

and

$$
P_{S, i, j}:=\bigcup_{f \in \Delta_{S, i, j,(i j)}}\left\{\left(e_{i}, f\right),\left(e_{j}, f\right)\right\} .
$$


In addition, for each $k \in S \backslash\{i, j\}$, let

$$
\Xi_{S, i, j, k}:=\bigcup_{\left(i^{\prime} j^{\prime}\right) \in \Gamma_{\{i, j, k\}}^{2}} \bigcup_{f \in \Delta_{S, i, j,\left(i^{\prime} j^{\prime}\right)}}\left\{\left(e_{i^{\prime}}, f\right),\left(e_{j^{\prime}}, f\right)\right\} .
$$

Proposition 5.8 Every cell of $\mathscr{S}$ is of the form $C \cup D$, where $D$ is a simplex affinely independent to $C$ and $C$ is a face of one of the following.

(a) $\Pi_{S, i, j} \cup \Xi_{S^{\prime}, i, j, k} \cup P_{S^{\prime \prime}, i, j}$ where $S, S^{\prime}, S^{\prime \prime} \in\left(\begin{array}{c}{[5]} \\ 4\end{array}\right)$ are distinct and contain $i, j$ and $\{k\}=[5] \backslash S$.

(b) $\Xi_{S, i, j, k} \cup \Xi_{S^{\prime}, i, j, k^{\prime}} \cup \Xi_{S^{\prime \prime}, i, j, k^{\prime \prime}}$ where $S, S^{\prime}, S^{\prime \prime} \in\left(\begin{array}{c}{[5]} \\ 4\end{array}\right)$ are distinct and contain $i, j$ and $k \in S \backslash\{i, j\}, k^{\prime} \in S^{\prime} \backslash\{i, j\}$, and $k^{\prime \prime} \in S^{\prime \prime} \backslash\{i, j\}$ are distinct.

We will call the cells in (a) and (b) the complex cells of $\mathscr{S}$.

Proof Using the notation of Theorem 3.3, the cell in (a) corresponds to $x \in \mathbb{R}^{5}$ with $x_{i}=0, x_{j}=1, x_{l}=\epsilon_{S, i, j, l}$ for each $l \in S \backslash\{i, j\}$, and $x_{k}=\epsilon_{S^{\prime}, i, j, k}$. The cell in (b) corresponds to $x \in \mathbb{R}^{5}$ with $x_{i}=0, x_{j}=1, x_{k}=\epsilon_{S, i, j, k}, x_{k^{\prime}}=\epsilon_{S^{\prime}, i, j, k^{\prime}}$, and $x_{k^{\prime \prime}}=\epsilon_{S^{\prime \prime}, i, j, k^{\prime \prime}}$. Due to the genericness of the $\epsilon_{S, i, j, k}$, it can be checked that every cell of $\mathscr{S}$ is the union of a face of one of these cells and an affinely independent simplex; we leave the details to the reader.

Thus, in order to give a triangulation of $\Pi$ which refines $\mathscr{S}$, it suffices to specify triangulations of the complex cells of $\mathscr{S}$ which agree on common faces. To do this, we first specify triangulations of each $\Pi_{S, i, j}$. We will then use a "pseudoproduct" operation to extend these to triangulations of the complex cells.

Fix $S \in\left(\begin{array}{c}{[5]} \\ 4\end{array}\right)$ and distinct $i, j \in S$. Let $\widetilde{\Pi}$ be the large 3-permutohedron defined in equation (4.3). Let $\psi:$ [4] $\rightarrow S$ be a map such that $\psi(1)=i$ and $\psi(2)=j$. We now choose the sizes of the $\Delta_{S, i, j,\left(i^{\prime} j^{\prime}\right)}$ so that we have an affine isomorphism $\Psi: \widetilde{\Pi} \rightarrow \Pi_{S, i, j}$ given by $e_{k} \mapsto e_{\psi(k)}$ for all $k \in[4]$ and so that $\left\{f_{k l}^{r}\right\}_{-N \leq r \leq N}$ maps bijectively to $\Delta_{S, i, j,(\psi(k) \psi(l))}$. We will define $\Psi: \widetilde{\Pi} \rightarrow \Pi_{S, i, j}$ so that $f_{12}^{-N}$ maps to $f_{i, j}^{*}$.

Let $\widetilde{\mathscr{T}}$ and $\widetilde{\mathscr{C}}$ be the triangulation of $\widetilde{\Pi}$ and the collection of triangulations, respectively, constructed in Sect. 4.4 which satisfy Proposition 4.10. Let $\mathscr{T}_{S, i, j}$ and $\widetilde{\mathscr{C}}_{S, i, j}$ be the images of $\widetilde{\mathscr{T}}$ and $\widetilde{\mathscr{C}}$, respectively, under $\Psi$. We thus have a triangulation $\mathscr{T}_{S, i, j}$ of each $\Pi_{S, i, j}$.

To extend the $\mathscr{T}_{S, i, j}$ to triangulations of the complex cells, we use the following "ordered pseudoproduct" construction. It is proved in the Appendix.

Proposition 5.9 Let $\Pi_{1}, \Pi_{2}, \ldots, \Pi_{N}$, and $\rho$ be cells of $\Pi$ such that $\rho=$ $\left\{\left(e_{i}, f\right),\left(e_{j}, f\right)\right\}$ for some distinct $i, j \in[5]$ and $f \in \Delta_{(i j)}$, and for any $1 \leq r, s \leq N$, we have $G\left(\Pi_{r}\right) \cap G\left(\Pi_{s}\right)=G(\sigma)$. Let $\mathscr{T}_{1}, \ldots, \mathscr{T}_{N}$ be triangulations of $\Pi_{1}, \ldots, \Pi_{N}$, respectively. Let $\mathscr{M}$ be the set of all simplices $\sigma \in A$ of the following form: There is an integer $1 \leq s \leq N$ such that

$$
\sigma=\left(\bigcup_{r<s}\left(\sigma_{r} \backslash \rho\right)\right) \cup \sigma_{s} \cup\left(\bigcup_{r>s} \sigma_{r}\right)
$$


where

- If $r<s$, then $\sigma_{r}$ is a maximal simplex of $\mathscr{T}_{r}$ with $\rho \subseteq \sigma_{r}$.

- $\sigma_{s}$ is a maximal simplex of $\mathscr{T}_{s}$, and if $s<N$, then $\rho^{\prime}:=\sigma_{s} \cap \rho \neq \rho$.

- If $r>s$, then $\sigma_{r}$ is a maximal simplex of $\mathscr{T}_{r}\left[F_{r}\right]$, where $F_{r}$ is a facet of $\Pi_{r}$ with $F_{r} \cap \rho=\rho^{\prime}$.

Then $\mathscr{M}$ is the set of maximal simplices of a triangulation $\mathscr{T}\left(\mathscr{T}_{1}, \ldots, \mathscr{T}_{N}\right)$ of $\Pi_{1} \cup$ $\cdots \cup \Pi_{N}$, and $\mathscr{T}_{r} \subseteq \mathscr{T}\left(\mathscr{T}_{1}, \ldots, \mathscr{T}_{N}\right)$ for all $1 \leq r \leq N$.

Now, fix distinct $i, j \in$ [5], and specify an ordering $S_{1}, S_{2}, S_{3}$ of the sets $S \in\left(\begin{array}{c}{[5]} \\ 4\end{array}\right)$ which contain $i$ and $j$. Each complex cell is of the form $F_{1} \cup F_{2} \cup F_{3}$ where for each distinct $1 \leq r, s \leq 3, F_{r}$ is a face of $\Pi_{S_{r}, i, j}$, and $G\left(F_{r}\right) \cap G\left(F_{s}\right)=G(\rho)$ where

$$
\rho:=\left\{\left(e_{i}, f_{i, j}^{*}\right),\left(e_{j}, f_{i, j}^{*}\right)\right\}
$$

By Proposition 5.9, we thus have a triangulation $\mathscr{T}\left(\mathscr{T}_{S_{1}, i, j}\left[F_{1}\right], \mathscr{T}_{S_{2}, i, j}\left[F_{2}\right], \mathscr{T}_{S_{3}, i, j}\right.$ $\left.\left[F_{3}\right]\right)$ of $F_{1} \cup F_{2} \cup F_{3}$. We leave it as an exercise to verify that all of these triangulations of the complex cells agree on common faces. ${ }^{1}$ Hence, we can extend these triangulations of the complex cells to a triangulation $\mathscr{T}$ of $\Pi$. By Proposition 5.9, this triangulation $\mathscr{T}$ contains all the triangulations $\mathscr{T}_{S, i, j}$ as subcollections.

For each $S \in\left(\begin{array}{c}{[5]} \\ 4\end{array}\right)$ and distinct $i, j \in[n]$, let $\mathscr{D}_{S, i, j}$ be the set of all zonotopal triangulations of the form $\mathscr{T}_{i j k}^{f_{1} f_{2} f_{3}}$ such that $k \in S \backslash\{i, j\}$ and $\mathscr{T}_{i j k}^{f_{i, j}^{*} f_{2} f_{3}} \in \tilde{\mathscr{C}}_{S, i, j}$. Let

$$
\mathscr{C}_{S, i, j}:=\tilde{\mathscr{C}}_{S, i, j} \cup \mathscr{D}_{S, i, j}
$$

We now finally show that the assumptions of Lemma 5.2 hold for $\mathscr{C}_{S, i, j}$ and $\mathscr{T}$.

Proposition 5.10 The collections $\mathscr{C}_{S, i, j}$ satisfy Properties 1-3 of Lemma 5.2.

Proof We first prove that Properties 1 and 2 hold. Suppose $\mathscr{T}_{i_{1} i_{2} i_{3}}^{f_{1} f_{2} f_{3}} \in \mathscr{C}_{S, i, j}$. If $\mathscr{T}_{i_{1} i_{2} i_{3}}^{f_{1} f_{2} f_{3}} \in \widetilde{\mathscr{C}}_{S, i, j}$, then Properties 1 and 2 follow from Proposition 4.10 (B) by setting the appropriate values of $\gamma$. So we may assume $\mathscr{T}_{i_{1} i_{2} i_{3}}^{f_{1} f_{3} f_{3}}=\mathscr{T}_{i j k}^{f_{1} f_{2} f_{3}} \in \mathscr{D}_{S, i, j}$. By definition of $\mathscr{D}_{S, i, j}$, we have $\mathscr{T}_{i j k}^{f_{i, j}^{*} f_{2} f_{3}} \in \widetilde{\mathscr{C}}_{S, i, j}$. Hence, by Proposition $4.10(\mathrm{~B})$, there exist $f_{1}^{\prime} \in \Delta_{S, i, j,(i l)}, f_{2}^{\prime} \in \Delta_{S, i, j,(j l)}$, and $f_{3}^{\prime} \in \Delta_{S, i, j,(k l)}$, where $l=S \backslash\{i, j, k\}$, such that

$$
\mathscr{T}_{i j l}^{f_{i, j}^{*} f_{2}^{\prime} f_{1}^{\prime}}, \mathscr{T}_{j k l}^{f_{2} f_{3}^{\prime} f_{2}^{\prime}}, \mathscr{T}_{k i l}^{f_{3} f_{1}^{\prime} f_{3}^{\prime}} \in \widetilde{\mathscr{C}}_{S, i, j}
$$

By definition, we thus have $\mathscr{T}_{i j l}^{f_{1} f_{2}^{\prime} f_{1}^{\prime}} \in \mathscr{D}_{S, i, j}$. Hence

$$
\mathscr{T}_{i j l}^{f_{1} f_{2}^{\prime} f_{1}^{\prime}}, \mathscr{T}_{j k l}^{f_{2} f_{3}^{\prime} f_{2}^{\prime}}, \mathscr{T}_{k i l}^{f_{3} f_{1}^{\prime} f_{3}^{\prime}} \in \mathscr{C}_{S, i, j}
$$

\footnotetext{
1 The argument is the same as the proof of Property 1 in the proof Proposition 5.9.
} 
which proves Property 1 . The argument for Property 2 is analogous.

We now prove Property 3. Let $i, j, l \in[5]$ be distinct and let $f_{1} \in \Delta_{(i j)}$. Let $S=[5] \backslash\{l\}$. By Proposition 4.10(A) applied to the triangulation $\mathscr{T}_{S, i, j}$, for any $k \in S \backslash\{i, j\}$ there exists $f_{2} \in \Delta_{S, i, j,(j k)}$ and $f_{3} \in \Delta_{S, i, j,(k i)}$ such that

$$
\mathscr{T}_{i j k}^{f_{i, j}^{*} f_{2} f_{3}} \in \tilde{\mathscr{C}}_{S, i, j}
$$

Thus by definition, $\mathscr{T}_{i j k}^{f_{1} f_{2} f_{3}} \in \mathscr{D}_{S, i, j} \subseteq \mathscr{C}_{S, i, j}$, which proves Property 3 .

Proposition 5.11 Properties (i) and (ii) of Lemma 5.2 hold for $\mathscr{T}$ with respect to the collections $\mathscr{C}_{S, i, j}$.

Proof We first note the following two facts.

Proposition 5.12 For any $f \in \Delta_{(i j)}$, we have $\left\{\left(e_{i}, f_{i, j}^{*}\right),\left(e_{j}, f\right)\right\} \in \mathscr{T}$.

Proof In our construction of $\mathscr{S}$ from $\omega$, we had

$$
\omega\left(e_{i}, f_{i, j}^{*}\right)-\omega\left(e_{j}, f_{i, j}^{*}\right)+\omega\left(e_{j}, f\right)-\omega\left(e_{i}, f\right) \leq 0
$$

for all $f \in \Delta_{(i j)}$, with equality if and only if $f \in \Delta_{i, j}$. In addition, $\widetilde{\mathscr{T}}$ is a refinement of a regular subdivision of $\widetilde{\Pi}$ given by height function $\widetilde{\omega}$ where

$$
\widetilde{\omega}\left(e_{1}, f_{12}^{-N}\right)-\widetilde{\omega}\left(e_{2}, f_{12}^{-N}\right)+\widetilde{\omega}\left(e_{2}, f_{12}^{r}\right)-\widetilde{\omega}\left(e_{1}, f_{12}^{r}\right)<0
$$

for all $r \neq N$. Thus, for all $f \in \Delta_{(i j)}$, restricting $\mathscr{T}$ to the face $X_{i j}^{f_{i, j}^{*} f}$ of $\Pi$ yields the triangulation $\mathscr{T}_{X_{i j}^{*} f_{j}^{*} f}^{-}$, and hence $\left\{\left(e_{i}, f_{i, j}^{*}\right),\left(e_{j}, f\right)\right\} \in \mathscr{T}$.

Proposition 5.13 Let $\mathscr{T}_{i j k}^{f_{i, j}^{*} f_{2} f_{3}} \subseteq \mathscr{T}$ and $f_{1} \in \Delta_{(i j)}$. Then $\mathscr{T}_{i j k}^{f_{1} f_{2} f_{3}} \subseteq \mathscr{T}$.

Proof Let

$$
\sigma_{0}:=X_{i j k}^{f_{i, j}^{*} f_{2} f_{3}} \backslash\left\{\left(e_{i}, f_{i, j}^{*}\right)\right\} \in \mathscr{T}
$$

By considering a maximal simplex of $\mathscr{T}$ containing $\sigma_{0}$, we have $\sigma_{0} \cup\left\{\left(e, f_{1}\right)\right\} \in \mathscr{T}$ for some $e \in\left\{e_{i}, e_{j}\right\}$. If $e=e_{i}$, then $\left\{\left(e_{i}, f_{1}\right),\left(e_{j}, f_{i, j}^{*}\right)\right\} \subseteq \sigma_{0}$. However, this contradicts Propositions 5.12 and 6.2 in the Appendix. Thus $e=e_{j}$. Hence, we have

$$
\sigma_{1}:=X_{i j k}^{f_{1} f_{2} f_{3}} \backslash\left\{\left(e_{i}, f_{1}\right)\right\} \in \mathscr{T}
$$

Now, the circuit $X:=X_{i j k}^{f_{1} f_{2} f_{3}}$ is a face of $\Pi$, so $\mathscr{T}[X]$ is a triangulation of $X$. Since $\sigma_{1} \in \mathscr{T}[X]$, this triangulation must be $\mathscr{T}_{X}^{+}$. Thus $\mathscr{T}_{i j k}^{f_{1} f_{2} f_{3}} \subseteq \mathscr{T}$. 
Now, for any $\mathscr{T}_{i_{1} i_{2} i_{3}}^{f_{1} f_{2} f_{3}} \in \tilde{\mathscr{C}}_{S, i, j}$, by definition of $\widetilde{\mathscr{T}}$ and $\tilde{\mathscr{C}}$ we have $\mathscr{T}_{i_{1} i_{2} i_{3}}^{f_{1} f_{2} f_{3}} \subseteq$ $\mathscr{T}_{S, i, j} \subseteq \mathscr{T}$. Suppose $\mathscr{T}_{i j k}^{f_{1} f_{2} f_{3}} \in \mathscr{D}_{S, i, j}$. Then $\mathscr{T}_{i j k}^{f_{i, j}^{*} f_{2} f_{3}} \in \tilde{\mathscr{C}}_{S, i, j}$ by definition. Thus $\mathscr{T}_{i j k}^{f_{i, j}^{*} f_{2} f_{3}} \subseteq \mathscr{T}$, so by Proposition 5.13, $\mathscr{T}_{i j k}^{f_{1} f_{2} f_{3}} \subseteq \mathscr{T}$. Hence Property (i) holds.

Now suppose $\mathscr{T}_{i j k}^{f_{1} f_{2} f_{3}} \in \mathscr{C}_{S, i, j}$, and let $f \in \Delta_{(i l)}$ where $\{l\}=[5] \backslash S$. By Property (i), we have $X_{i j k}^{f_{1} f_{2} f_{3}} \backslash\left\{\left(e_{i}, f_{1}\right)\right\} \in \mathscr{T}$. By Proposition 6.1 in the Appendix, we have $X_{i j k}^{f_{1} f_{2} f_{3}} \backslash\left\{\left(e_{i}, f_{1}\right)\right\} \cup\left\{\left(e_{i}, f\right)\right\} \in \mathscr{T}$. Thus Property (ii) holds.

Hence, we have collections $\mathscr{C}_{S, i, j}$ which satisfy the hypotheses of Lemma 5.2, and by Propositions 5.11 and 5.7, there exists a triangulation $\mathscr{T}^{\prime} \in \mathcal{S}_{\mathscr{C}}$. Clearly $\mathcal{S}_{\mathscr{C}}$ is not the set of all triangulations of $A$; for example, there exist triangulations of $A$ which do not contain any triangulations of circuits of size six [2]. Hence Lemma 5.2 proves Theorem 5.1.

\section{Appendix}

\subsection{Proof of Proposition 5.3}

Since $\sigma \notin \mathscr{T}^{\prime}$, by Proposition 2.2 we have $\sigma \supseteq X^{-}$. Let $\tau$ be a maximal simplex of $\mathscr{T}$ containing $\sigma$. We must have $\tau \notin \mathscr{T}^{\prime}$ since $\sigma \notin \mathscr{T}^{\prime}$. So by Proposition 2.2, $\tau$ must contain a maximal simplex $\tau^{\prime}$ of $\mathscr{T}_{X}^{+}$. Now, since $\sigma \cup \tau^{\prime} \subseteq \tau$, we have that $G\left(\sigma \cup \tau^{\prime}\right)$ is acyclic. But since $\sigma \supseteq X^{-}$and $G(\sigma)$ is connected, this can only happen if $\sigma \supseteq \tau^{\prime}$, as desired.

\subsection{Proof of Proposition 5.4}

Throughout this section, let $A:=\Delta^{m-1} \times \Delta^{n-1}$. We need the following two facts.

Proposition 6.1 Let $\mathscr{T}$ be a triangulation of A. Let $\sigma \in \mathscr{T}$. Suppose that $f \in \Delta^{n-1}$ and $f \notin G(\sigma)$. Then there exists $e \in \Delta^{m-1} \cap G(\sigma)$ such that $\sigma \cup\{(e, f)\} \in \mathscr{T}$.

Proof Let $\Delta_{\sigma}:=\Delta^{m-1} \cap G(\sigma)$. Let $\phi: A \rightarrow \mathbb{R}$ be the linear functional with

$$
\begin{gathered}
\phi(e, 0)= \begin{cases}-1 & \text { if } e \in \Delta_{\sigma} \\
0 & \text { otherwise. }\end{cases} \\
\phi(0, f)=0 \text { for all } f \in \Delta^{n-1} .
\end{gathered}
$$

Let $F$ be the face of $A$ which minimizes $\phi$. Then $\sigma \subseteq F$. Let $\tau$ be a maximal simplex of $\mathscr{T}[F]$ containing $\sigma$. Then $\tau \supseteq \sigma \cup\{(e, f)\}$ for some $e \in \Delta_{\sigma}$.

By symmetry of $\Delta^{m-1}$ and $\Delta^{n-1}$, the above proposition also holds after replacing $f \in \Delta^{n-1}$ with $e \in \Delta^{m-1}$.

Proposition 6.2 Let $\mathscr{T}$ be a triangulation of $A$, and suppose $X=\left(X^{+}, X^{-}\right)$is a circuit of $A$. Then $\mathscr{T}$ does not contain both $X^{+}$and $X^{-}$. 
Fig. $3 G\left(\sigma_{0}\right)$

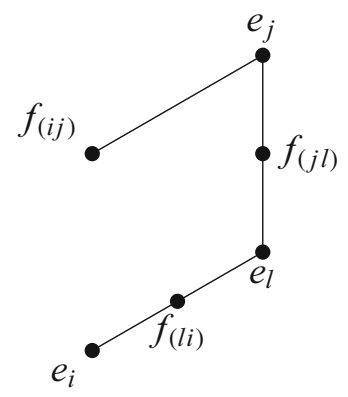

Proof The interiors of the convex hulls of opposite parts of a circuit intersect.

The next proposition immediately implies Proposition 5.4.

Proposition 6.3 Let $\mathscr{T}$ be a triangulation of A. Let $i, j, k, l \in[m]$ be distinct, and for each $\alpha \in \Gamma_{\{i, j, k, l\}}^{2}$, let $f_{\alpha} \in \Delta^{n-1}$. Suppose that

$$
\mathscr{T}_{j k l}^{f_{(j k)} f_{(k l)} f_{(l j)}}, \mathscr{T}_{k i l}^{f_{(k i)} f_{(i l)} f_{(l k)}} \subseteq \mathscr{T}
$$

and

$$
X_{i j l}^{f_{(i j)} f_{(j l)} f_{(l i)}} \backslash\left\{\left(e_{i}, f_{(i j)}\right)\right\} \in \mathscr{T} .
$$

Then $\mathscr{T}$ does not have a flip supported on $X_{i j k}^{f_{(i j)} f_{(j k)} f_{(k i)}}$.

Proof Suppose the contrary. In particular, this means

$$
\mathscr{T}_{i j k}^{f_{(i j)} f_{(j k)} f_{(k i)}} \subseteq \mathscr{T}
$$

Set $\sigma_{0}=X_{i j l}^{f_{(i j)} f_{(j l)} f_{(l i)}} \backslash\left\{\left(e_{i}, f_{(i j)}\right)\right\}$ (Fig. 3). By assumption, $\sigma_{0} \in \mathscr{T}$. By Proposition 6.1, there exists $\sigma_{1} \in \mathscr{T}$ such that

$$
\sigma_{1}=\sigma_{0} \cup\left\{\left(e, f_{(j k)}\right)\right\} \cup\left\{\left(e^{\prime}, f_{(k l)}\right)\right\},
$$

where $e, e^{\prime} \in\left\{e_{i}, e_{j}, e_{l}\right\}$. We prove the following claims.

Claim 1: $e=e_{j}$.

Suppose first that $e=e_{i}$. Then $\left\{\left(e_{i}, f_{(j k)}\right),\left(e_{j}, f_{(i j)}\right)\right\} \subseteq \sigma_{1}$. However, we also

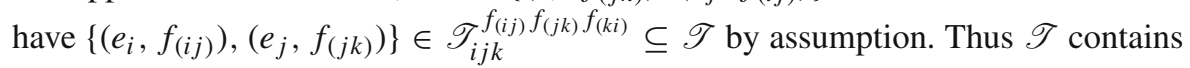
both parts of the circuit $X_{i j}^{f_{(j k)} f_{(i j)}}$, which contradicts Proposition 6.2.

Now suppose that $e=e_{l}$. Then $\left\{\left(e_{l}, f_{(j k)}\right),\left(e_{j}, f_{(j l)}\right)\right\} \subseteq \sigma_{1}$. However, we also have $\left\{\left(e_{l}, f_{(j l)}\right),\left(e_{j}, f_{(j k)}\right)\right\} \in \mathscr{T}_{j k l}^{f_{(j k)} f_{(k l)} f_{(l j)}} \subseteq \mathscr{T}$, again a contradiction. So $e=e_{j}$. 
Fig. $4 G\left(\sigma_{1}\right)$

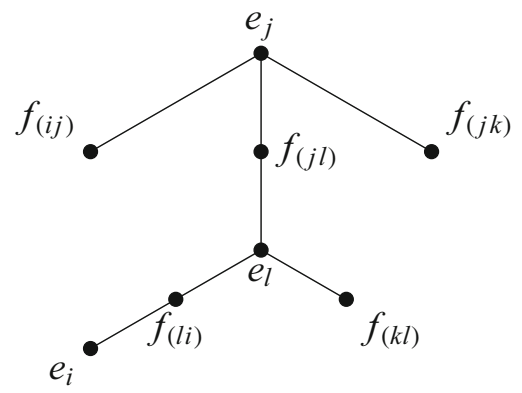

Fig. $5 G\left(\sigma_{2}\right)$

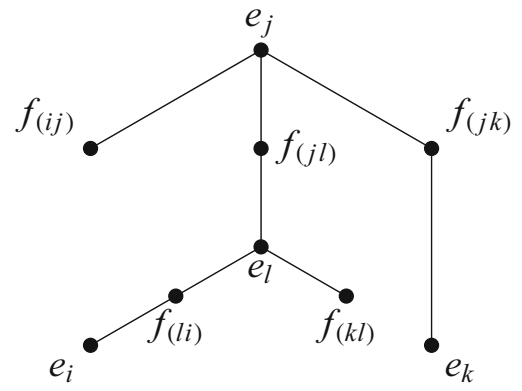

Claim 2: $e^{\prime}=e_{l}$.

Suppose first that $e^{\prime}=e_{i}$. Then $\left\{\left(e_{i}, f_{(k l)}\right),\left(e_{l}, f_{(l i)}\right)\right\} \subseteq \sigma_{1}$. However, we have $\left\{\left(e_{i}, f_{(l i)}\right),\left(e_{l}, f_{(k l)}\right)\right\} \in \mathscr{T}_{k i l}^{f_{(k i)} f_{(i l)} f_{(l k)}} \subseteq \mathscr{T}$, a contradiction.

Now suppose that $e^{\prime}=e_{j}$. Then $\left\{\left(e_{j}, f_{(k l)}\right),\left(e_{l}, f_{(j l)}\right)\right\} \subseteq \sigma_{1}$. However, we have $\left\{\left(e_{j}, f_{(j l)}\right),\left(e_{l}, f_{(k l)}\right)\right\} \in \mathscr{T}_{j k l}^{f_{(j k)} f_{(k l)} f_{(l j)}} \subseteq \mathscr{T}$, a contradiction. So $e^{\prime}=e_{l}$ (Fig. 4).

Now, by Proposition 6.1 and the comment afterwards, there exists $\sigma_{2} \in \mathscr{T}$ such that

$$
\sigma_{2}=\sigma_{1} \cup\left\{\left(e_{k}, f\right)\right\}
$$

where $f \in\left\{f_{(i j)}, f_{(j k)}, f_{(i l)}, f_{(j l)}, f_{(k l)}\right\}$. We prove the following.

Claim 3: $f=f_{(j k)}$.

The argument goes as follows:

If $f=f_{(i j)}$, then $\left\{\left(e_{k}, f_{(i j)}\right),\left(e_{j}, f_{(j k)}\right)\right\} \subseteq \sigma_{2}$ but $\left\{\left(e_{k}, f_{(j k)}\right),\left(e_{j}, f_{(i j)}\right)\right\} \in$ $\mathscr{T}_{i j k}^{f_{(i j)} f_{(j k)} f_{(k i)}}$.

If $f=f_{(i l)}$, then $\left\{\left(e_{k}, f_{(i l)}\right),\left(e_{l}, f_{(k l)}\right)\right\} \subseteq \sigma_{2}$ but $\left\{\left(e_{k}, f_{(k l)}\right),\left(e_{l}, f_{(i l)}\right)\right\} \in$ $\mathscr{T}_{k i l}^{f_{(k i)} f_{(i l)} f_{(l k)}}$.

If $f=f_{(j l)}$, then $\left\{\left(e_{k}, f_{(j l)}\right),\left(e_{j}, f_{(j k)}\right)\right\} \subseteq \sigma_{2}$ but $\left\{\left(e_{k}, f_{(j k)}\right),\left(e_{j}, f_{(j l)}\right)\right\} \in$ $\mathscr{T}_{j k l}^{f_{(j k)} f_{(k l)} f_{(l j)}}$.

If $f=f_{(k l)}$, then $\left(X_{j k l}^{f_{(j k)} f_{(k l)} f_{(l j)}}\right)^{+} \subseteq \sigma_{2}$, but $\left(X_{j k l}^{f_{(j k)} f_{(k l)} f_{(l j)}}\right)^{-} \in \mathscr{T}_{j k l}^{f_{(j k)} f_{(k l)} f_{(l j)}}$.

Thus $f=f_{(j k)}$ (Fig. 5). 
Fig. $6 G\left(\sigma_{3}\right)$

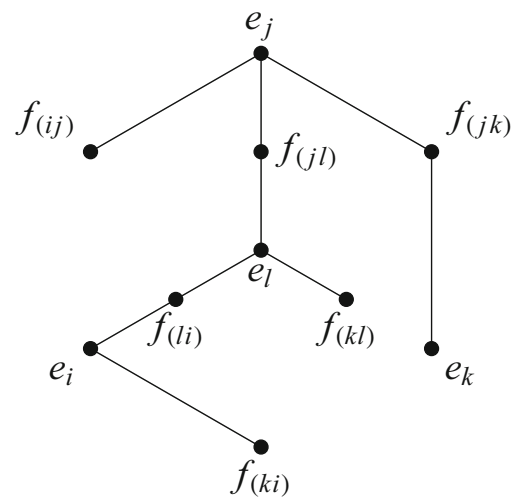

Finally, by Proposition 6.1 there exists $\sigma_{3} \in \mathscr{T}$ such that

$$
\sigma_{3}=\sigma_{2} \cup\left\{\left(e, f_{(k i)}\right)\right\}
$$

where $e \in\left\{e_{i}, e_{j}, e_{k}, e_{l}\right\}$.

Claim 4: $e=e_{i}$.

The argument goes as follows.

If $e=e_{j}$, then $\left\{\left(e_{j}, f_{(k i)}\right),\left(e_{k}, f_{(j k)}\right)\right\} \subseteq \sigma_{3}$ but $\left\{\left(e_{j}, f_{(j k)}\right),\left(e_{k}, f_{(k i)}\right)\right\} \in$ $\mathscr{T}_{i j k}^{f_{(i j)} f_{(j k)} f_{(k i)}}$.

If $e=e_{k}$, then $\left(X_{k i l}^{f_{(k i)} f_{(i l)} f_{(l k)}}\right)^{+} \subseteq \sigma_{3}$ but $\left(X_{k i l}^{f_{(k i)} f_{(i l)} f_{(l k)}}\right)^{-} \in \mathscr{T}_{k i l}^{f_{(k i)} f_{(i l)} f_{(l k)}}$.

If $e=e_{l}$, then $\left\{\left(e_{l}, f_{(k i)}\right),\left(e_{i}, f_{(i l)}\right)\right\} \subseteq \sigma_{3}$ but $\left\{\left(e_{l}, f_{(i l)}\right),\left(e_{i}, f_{(k i)}\right)\right\} \in$ $\mathscr{T}_{k i l}^{f_{(k i)} f_{(i l)} f_{(l k)}}$.

Thus $e=e_{i}$ (Fig. 6).

Now, let $\tau$ be a maximal simplex of $\mathscr{T}$ containing $\sigma_{3}$. Then $\left(X_{i j k}^{f_{(i j)} f_{(j k)} f_{(k i)}}\right)^{-} \subseteq \tau$ but $\left|X_{i j k}^{f_{(i j)} f_{(j k)} f_{(k i)}} \cap \tau\right|=4$. This contradicts Proposition 2.3 and our assumption that $\mathscr{T}$ has a flip supported on $X_{i j k}^{f_{(i j)} f_{(j k)} f_{(k i)}}$. So $\mathscr{T}$ does not have such a flip.

\subsection{Proof of Proposition 5.9}

Our proof is based on the following.

Theorem 6.4 (Rambau [8]) Let $\mathscr{M}$ be a nonempty collection of full-dimensional simplices of a point set $A$. Then $\mathscr{M}$ is the set of maximal simplices of a triangulation of $A$ if and only if

1. There is no circuit $X=\left(X^{+}, X^{-}\right)$of $A$ and simplices $\tau, \tau^{\prime} \in \mathscr{M}$ such that $X^{+} \subseteq \tau, X^{-} \subseteq \tau^{\prime}$.

2. For any simplex $\tau \in \mathscr{M}$ and facet $\sigma$ of $\tau$, either $\sigma$ is contained in a facet of $A$ or there is another $\tau^{\prime} \in \mathscr{M}, \tau^{\prime} \neq \tau$, such that $\sigma \subseteq \tau^{\prime}$. 
Let $\mathscr{M}$ be as in Proposition 5.9. We prove Properties 1 and 2 of Theorem 6.4 for $\mathscr{M}$.

Proof of Property 1 Suppose that $X=\left(X^{+}, X^{-}\right)$is a circuit of $\Pi_{1} \cup \cdots \cup \Pi_{N}$ and we have $X^{+} \subseteq \sigma, X^{-} \subseteq \sigma^{\prime}$ for some $\sigma, \sigma^{\prime} \in \mathscr{M}$. Let

$$
\begin{gathered}
\sigma=\left(\bigcup_{r<s}\left(\sigma_{r} \backslash \rho\right)\right) \cup \sigma_{s} \cup\left(\bigcup_{r>s} \sigma_{r}\right), \\
\sigma^{\prime}=\left(\bigcup_{r<s^{\prime}}\left(\sigma_{r}^{\prime} \backslash \rho\right)\right) \cup \sigma_{s^{\prime}}^{\prime} \cup\left(\bigcup_{r>s^{\prime}} \sigma_{r}^{\prime}\right)
\end{gathered}
$$

as in Proposition 5.9, with analogous definitions for $\sigma^{\prime}$. Suppose first that $X \subseteq \Pi_{r}$ for some $1 \leq r \leq N$. Then we have $X^{+} \subseteq \sigma \cap \Pi_{r} \subseteq \sigma_{r}$ and similarly $X^{-} \subseteq \sigma_{r}^{\prime}$. This contradicts the fact that $\sigma_{r}, \sigma_{r}^{\prime}$ are simplices of a triangulation $\mathscr{T}_{r}$. So we cannot have this case. It follows that $X \subseteq \Pi_{r_{1}} \cup \Pi_{r_{2}}$ for some $1 \leq r_{1}<r_{2} \leq N$, and we can uniquely write

$$
X=X_{r_{1}} \cup X_{r_{2}} \backslash \rho,
$$

where $X_{r_{1}}=\left(X_{r_{1}}^{+}, X_{r_{1}}^{-}\right)$and $X_{r_{2}}=\left(X_{r_{2}}^{+}, X_{r_{2}}^{-}\right)$are circuits in $\Pi_{r_{1}}$ and $\Pi_{r_{2}}$ respectively, and

$$
\begin{array}{ll}
X_{r_{1}}^{+}=\left(X^{+} \cap \Pi_{r_{1}}\right) \cup \rho_{1}, & X_{r_{1}}^{-}=\left(X^{-} \cap \Pi_{r_{1}}\right) \cup \rho_{2}, \\
X_{r_{2}}^{+}=\left(X^{+} \cap \Pi_{r_{2}}\right) \cup \rho_{2}, & X_{r_{2}}^{-}=\left(X^{-} \cap \Pi_{r_{2}}\right) \cup \rho_{1},
\end{array}
$$

where $\rho_{1}, \rho_{2}$ are different one-element subsets of $\rho$.

We claim that $X_{r_{1}}^{+} \subseteq \sigma_{r_{1}}$. First suppose that $r_{1}<s$. Then $\left(X^{+} \cap \Pi_{r_{1}}\right) \cup \rho \subseteq \sigma_{r_{1}}$, so $X_{r_{1}}^{+} \subseteq \sigma_{r_{1}}$, as desired. So we may assume $r_{1} \geq s$, and hence $s<r_{2} \leq N$. Now, since $s<N$, we have $\sigma \cap \rho=\rho_{i}$ for either $i=1$ or 2 . If $i=1$, then $X_{r_{1}}^{+} \subseteq \sigma_{r_{1}}$ and we are done. Suppose $i=2$. Then $X_{r_{2}}^{+} \subseteq \sigma_{r_{2}}$. Since $r_{2}>s$, it follows that $X_{r_{2}}^{+}$is contained in a facet $F$ of $\Pi_{r_{2}}$ with $F \cap \rho=\rho_{2}$. However, $X_{r_{2}}^{+} \subseteq F$ implies $X_{r_{2}} \subseteq F$ because $F$ is a face of $\Pi_{r_{2}}$. This contradicts $F \cap \rho=\rho_{2}$. Hence we have $X_{r_{1}}^{+} \subseteq \sigma_{r_{1}}$.

By the same argument, we have $X_{r_{1}}^{-} \subseteq \sigma_{r_{1}}^{\prime}$. Hence $X_{r_{1}}^{+}, X_{r_{1}}^{-} \in \mathscr{T}_{r_{1}}$, a contradiction. This proves Property 1.

Proof of Property 2 Let

$$
\sigma=\left(\bigcup_{r<s}\left(\sigma_{r} \backslash \rho\right)\right) \cup \sigma_{s} \cup\left(\bigcup_{r>s} \sigma_{r}\right)
$$

be an element of $\mathscr{M}$ as before. Let $x \in \sigma$, and consider the facet $\sigma \backslash\{x\}$. We have the following cases. 
Case 1: $x \in \sigma_{t} \backslash \rho$ where $t<s$.

Subcase 1.1: $\sigma_{t} \backslash\{x\}$ is not contained in a facet of $\Pi_{t}$. Then there is some $x^{\prime} \in \Pi_{t}$ such that $\sigma_{t}^{\prime}:=\sigma_{t} \backslash\{x\} \cup\left\{x^{\prime}\right\} \in \mathscr{T}_{t}$. Then

$$
\sigma \backslash\{x\} \cup\left\{x^{\prime}\right\}=\left(\bigcup_{\substack{r<s \\ r \neq t}}\left(\sigma_{r} \backslash \rho\right)\right) \cup\left(\sigma_{t}^{\prime} \backslash \rho\right) \cup \sigma_{s} \cup\left(\bigcup_{r>s} \sigma_{r}\right)
$$

is an element of $\mathscr{M}$ containing $\sigma \backslash\{x\}$, as desired.

Subcase 1.2: $\sigma_{t} \backslash\{x\}$ is contained in a facet $F$ of $\Pi_{t}$. Let $\phi: \Pi_{t} \rightarrow \mathbb{R}$ be a linear functional supporting $F$ on $\Pi_{t}$. Since $\rho \subseteq \sigma_{t} \backslash\{x\}$, we have $\rho \subseteq F$, hence $\phi(\rho)=\{b\}$ where $b$ is the minimum of $\phi$ on $\Pi_{t}$. We may assume $b=0 .^{2}$ Now, we can extend $\phi$ to a linear functional $\phi^{\prime}$ on $\Pi_{1} \cup \cdots \cup \Pi_{N}$ by setting $\phi^{\prime}(v)=\phi(v)$ for all $v \in \Pi_{t}$ and $\phi^{\prime}(v)=0$ for all $v \in \Pi_{r}, r \neq t .^{3}$ Then the face of $\Pi_{1} \cup \cdots \cup \Pi_{N}$ supported by $\phi^{\prime}$ contains $\sigma \backslash\{x\}$, and this face is proper because it does not contain $\Pi_{t}$. Thus $\sigma \backslash\{x\}$ is contained in a facet of $\Pi_{1} \cup \cdots \cup \Pi_{N}$, as desired.

Case 2: $x \in \rho$.

Subcase 2.1: $\sigma \cap \rho \neq \rho$. Then $\sigma \backslash\{x\}$ is contained in a facet of $\Pi_{1} \cup \cdots \cup \Pi_{N}$ because $f$ has no neighbors in $G(\sigma \backslash\{x\})$.

Subcase 2.2: $\sigma \cap \rho=\rho$. This implies $s=N$ and $x \in \sigma_{r}$ for all $r$. First, suppose there is some $t$ such that $\sigma_{t} \backslash\{x\}$ is not contained in a facet of $\Pi_{t}$, and let $t$ be the largest such number. Then there is some $x^{\prime} \in \Pi_{t}$ such that $\sigma_{t}^{\prime}:=\sigma_{t} \backslash\{x\} \cup\left\{x^{\prime}\right\} \in \mathscr{T}_{t}$. Thus

$$
\sigma \backslash\{x\} \cup\left\{x^{\prime}\right\}=\left(\bigcup_{r<t}\left(\sigma_{r} \backslash \rho\right)\right) \cup \sigma_{t}^{\prime} \cup\left(\bigcup_{r>t}\left(\sigma_{r} \backslash\{x\}\right)\right)
$$

is an element of $\mathscr{M}$ containing $\sigma \backslash\{x\}$, as desired. ${ }^{4}$

Now suppose there is no such $t$. Then for all $r, \sigma_{r} \backslash\{x\}$ is contained in a facet $F_{r}$ of $\Pi_{r}$. We have $x \notin F_{r}$ since $\sigma_{r}$ is full dimensional in $\Pi_{r}$. For each $r$, let $\phi_{r}: \Pi_{r} \rightarrow \mathbb{R}$ be a linear functional supporting $F_{r}$ on $\Pi_{r}$. Let $\{y\}=\rho \backslash\{x\}$. Then $y \in F_{r}$ and $x \notin F_{r}$, so $\phi_{r}(y)<\phi_{r}(x)$. By appropriately choosing $\phi_{r}$, we may assume $\phi_{r}(y)=0$ and $\phi_{r}(x)=1$ for all $r$. As before, we can then define a linear functional $\phi^{\prime}$ on $\Pi_{1} \cup \cdots \cup \Pi_{N}$ such that $\phi^{\prime}(v)=\phi_{r}(v)$ for all $v \in \Pi_{r}$. Then the face of $\Pi_{1} \cup \cdots \cup \Pi_{N}$ supported by $\phi^{\prime}$ contains $\sigma \backslash\{x\}$, and this face is proper. Thus $\sigma \backslash\{x\}$ is contained in a facet of $\Pi_{1} \cup \cdots \cup \Pi_{N}$.

Case 3: $x \in \sigma_{s} \backslash \rho$.

Subcase 3.1: $\sigma_{s} \backslash\{x\}$ is not contained in a facet of $\Pi_{s}$. Then there is some $x^{\prime} \in \Pi_{s}$ such that $\sigma_{s}^{\prime}:=\sigma_{s} \backslash\{x\} \cup\left\{x^{\prime}\right\} \in \mathscr{T}_{s}$. First assume that $x^{\prime} \notin \rho$. Then

\footnotetext{
2 This is because $\Pi_{t}$ is contained in an affine subspace not containing the origin.

3 We can do this because any affine dependence in $\Pi_{1} \cup \cdots \cup \Pi_{N}$ can be written as a sum of affine dependencies each contained in one of the $\Pi_{r}$.

${ }^{4}$ In more detail: by definition of $t$, if $r>t$ then $\sigma_{r} \backslash\{x\}$ is contained in a facet $F$ of $\Pi_{r}$. We must have $F \cap \rho=\rho \backslash\{x\}$ because $\sigma_{r}$ is full-dimensional in $\Pi_{r}$.
} 


$$
\sigma \backslash\{x\} \cup\left\{x^{\prime}\right\}=\left(\bigcup_{r<s}\left(\sigma_{r} \backslash \rho\right)\right) \cup \sigma_{s}^{\prime} \cup\left(\bigcup_{r>s} \sigma_{r}\right)
$$

is an element of $\mathscr{M}$ containing $\sigma \backslash\{x\}$, as desired.

Now assume that $x^{\prime} \in \rho$. First, suppose that for all $r>s, \sigma_{r}^{\prime}:=\sigma_{r} \cup\left\{x^{\prime}\right\} \in \mathscr{T}_{r}$. Then

$$
\sigma \backslash\{x\} \cup\left\{x^{\prime}\right\}=\left(\bigcup_{r<s}\left(\sigma_{r} \backslash \rho\right)\right) \cup\left(\sigma_{s}^{\prime} \backslash \rho\right) \cup\left(\bigcup_{s<r<N}\left(\sigma_{r}^{\prime} \backslash \rho\right)\right) \cup \sigma_{N}^{\prime}
$$

is an element of $\mathscr{M}$ containing $\sigma \backslash\{x\}$, as desired. Now suppose that there is some $t>s$ such that $\sigma_{t} \cup\left\{x^{\prime}\right\} \notin \mathscr{T}_{t}$, and let $t$ be the smallest such number. Let $x^{\prime \prime}$ be a point in $\mathscr{T}_{t}$ such that $x^{\prime \prime} \notin \sigma_{t}$ and $\sigma_{t}^{\prime \prime}:=\sigma_{t} \cup\left\{x^{\prime \prime}\right\} \in \mathscr{T}_{t}$. By definition of $t, x^{\prime \prime} \neq x^{\prime}$, and hence $x^{\prime \prime} \notin \rho$. Then

$$
\sigma \backslash\{x\} \cup\left\{x^{\prime \prime}\right\}=\left(\bigcup_{r<s}\left(\sigma_{r} \backslash \rho\right)\right) \cup\left(\sigma_{s}^{\prime} \backslash \rho\right) \cup\left(\bigcup_{s<r<t}\left(\sigma_{r}^{\prime} \backslash \rho\right)\right) \cup \sigma_{t}^{\prime \prime} \cup\left(\bigcup_{r>t} \sigma_{r}\right)
$$

is an element of $\mathscr{M}$ containing $\sigma \backslash\{x\}$, as desired.

Subcase 3.2: $\sigma_{s} \backslash\{x\}$ is contained in a facet $F$ of $\Pi_{s}$. If $\rho \subseteq F$, then by the same argument as in Subcase 1.2, $\sigma \backslash\{x\}$ is contained in a facet of $\Pi_{1} \cup \cdots \cup \Pi_{N}$. So we may assume $\rho \nsubseteq F$. In particular, this means $\sigma_{s} \cap \rho=\rho \backslash\left\{x^{\prime}\right\}$ for some $x^{\prime} \in \rho$.

First, suppose that there is some $t<s$ such that $\sigma_{t} \backslash\left\{x^{\prime}\right\}$ is not contained in a facet of $\mathscr{T}_{t}$, and let $t$ be the largest such number. Then there is some $x^{\prime \prime} \in \mathscr{T}_{t}$ such that $\sigma_{t}^{\prime \prime}:=\sigma_{t} \backslash\left\{x^{\prime}\right\} \cup\left\{x^{\prime \prime}\right\} \in \mathscr{T}_{t}$. Then

$$
\sigma \backslash\{x\} \cup\left\{x^{\prime \prime}\right\}=\left(\bigcup_{r<t}\left(\sigma_{r} \backslash \rho\right)\right) \cup \sigma_{t}^{\prime \prime} \cup\left(\bigcup_{t<r<s}\left(\sigma_{r} \backslash\left\{x^{\prime}\right\}\right)\right) \cup\left(\sigma_{s} \backslash\{x\}\right) \cup\left(\bigcup_{r>s} \sigma_{r}\right)
$$

is an element of $\mathscr{M}$ containing $\sigma \backslash\{x\}$, as desired.

Now suppose that there is no such $t$. Then for all $r$, we have that $(\sigma \backslash\{x\}) \cap \Pi_{r}$ is contained in a facet $F_{r}$ of $\Pi_{r}$. We also have $F_{r} \cap \rho=\rho \backslash\left\{x^{\prime}\right\}$ for all $r$ : For $r<s$, this holds because $\sigma_{r}$ is maximal in $\Pi_{r}$, for $r=s$, this holds by our original assumption, and for $r>s$, this holds by definition. Hence, by the argument in the second part of Subcase 2.2, $\sigma \backslash\{x\}$ is contained in a facet of $\Pi_{1} \cup \cdots \cup \Pi_{N}$, as desired.

Case 4: $x \in \sigma_{t} \backslash \rho$ where $t>s$.

Let $\rho^{\prime}=\sigma_{t} \cap \rho$. Let $\mathscr{U} \subseteq \mathscr{T}_{t}$ be the simplicial complex consisting of all simplices in $\mathscr{T}_{t}$ on the boundary of $\Pi_{t}$. Let $\mathscr{U}^{\prime}$ be the subcomplex of $\mathscr{U}$ consisting of all simplices in $\mathscr{T}_{t}$ contained in facets $F$ of $\Pi_{t}$ with $F \cap \rho=\rho^{\prime}$. In particular, we have $\sigma_{t} \in \mathscr{U}^{\prime}$.

Since $\mathscr{U}$ is homeomorphic to a sphere, there is some $x^{\prime} \in \Pi_{t}$ such that $\sigma_{t}^{\prime}:=\sigma_{t} \backslash\{x\} \cup\left\{x^{\prime}\right\} \in \mathscr{U}$. If $\sigma_{t}^{\prime} \in \mathscr{U}^{\prime}$, then

$$
\sigma \backslash\{x\} \cup\left\{x^{\prime}\right\}=\left(\bigcup_{r<s}\left(\sigma_{r} \backslash \rho\right)\right) \cup \sigma_{s} \cup \sigma_{t}^{\prime} \cup\left(\bigcup_{\substack{r>s \\ r \neq t}} \sigma_{r}\right)
$$


is an element of $\mathscr{M}$ containing $\sigma \backslash\{x\}$, as desired. Suppose $\sigma_{t}^{\prime} \notin \mathscr{U}^{\prime}$. Let $F$ be the facet of $\Pi_{t}$ containing $\sigma_{t}^{\prime}$. Since $\rho^{\prime} \subseteq \sigma_{t}^{\prime}$, but $F \cap \rho \neq \rho^{\prime}$, we must have $\rho \subseteq F$. By the argument from Subcase 1.2, it follows that $\sigma \backslash\{x\}$ is contained in a facet of $\Pi_{1} \cup \cdots \cup \Pi_{N}$, as desired.

This proves that $\mathscr{M}$ is the set of maximal simplices of a triangulation $\mathscr{T}$. Suppose that $\sigma_{s}$ is a maximal simplex of $\mathscr{T}_{s}$. If $\rho \nsubseteq \sigma_{s}$, then for all $r \neq s$ we can choose $\sigma_{r} \in \Pi_{r}$ such that

$$
\left(\bigcup_{r<s}\left(\sigma_{r} \backslash \rho\right)\right) \cup \sigma_{s} \cup\left(\bigcup_{r>s} \sigma_{r}\right)
$$

is in $\mathscr{M}$. If $\rho \subseteq \sigma_{s}$, then for all $r \neq s$ we can choose $\sigma_{r} \in \Pi_{r}$ such that $\rho \subseteq \sigma_{r}$ for all $r$ and

$$
\sigma=\left(\bigcup_{r<s}\left(\sigma_{r} \backslash \rho\right)\right) \cup\left(\sigma_{s} \backslash \rho\right) \cup\left(\bigcup_{s<r<N}\left(\sigma_{r} \backslash \rho\right)\right) \cup \sigma_{N}
$$

is in $\mathscr{M}$. Either way, $\sigma_{s} \in \mathscr{T}$. Thus, $\mathscr{T}_{1}, \ldots, \mathscr{T}_{N} \subseteq \mathscr{T}$.

Acknowledgements Open access funding provided by Max Planck Society. The author would like to thank his advisor Alexander Postnikov and Francisco Santos for their valuable feedback and discussions. This material is based upon work supported by the National Science Foundation Graduate Research Fellowship under Grant No. 1122374.

Open Access This article is distributed under the terms of the Creative Commons Attribution 4.0 International License (http://creativecommons.org/licenses/by/4.0/), which permits unrestricted use, distribution, and reproduction in any medium, provided you give appropriate credit to the original author(s) and the source, provide a link to the Creative Commons license, and indicate if changes were made.

\section{References}

1. Billera, L.J., Kapranov, M.M., Sturmfels, B.: Cellular strings on polytopes. Proc. Am. Math. Soc. 122(2), 549-555 (1994)

2. De Loera, J.A., Rambau, J., Santos, F.: Triangulations: Structures for Algorithms and Applications. Algorithms and Computation in Mathematics. Springer, Berlin (2010)

3. Develin, M., Sturmfels, B.: Tropical convexity. Doc. Math. 9, 1-27 (2004)

4. Huber, B., Rambau, J., Santos, F.: The Cayley trick, lifting subdivisions and the Bohne-Dress theorem on zonotopal tilings. J. Eur. Math. Soc. 2(2), 179-198 (2000)

5. Kenyon, R.: Tiling a polygon with parallelograms. Algorithmica 9(4), 382-397 (1993)

6. Liu, G.: Flip-connectivity of triangulations of the product of a tetrahedron and simplex (2016). arXiv:1601.06031

7. Maclagan, D., Thomas, R.R.: Combinatorics of the toric Hilbert scheme. Discret. Comput. Geom. 27(2), 249-272 (2002)

8. Rambau, J.: Triangulations of cyclic polytopes and higher Bruhat orders. Mathematika 44(1), 162-194 (1997)

9. Reiner, V.: The generalized Baues problem. In: Billera, L.J., et al. (eds.) New Perspectives in Algebraic Combinatorics. MSRI Book Series, vol. 38, pp. 293-336. Cambridge University Press, New York (1999)

10. Santos, F.: A point set whose space of triangulations is disconnected. J. Am. Math. Soc. 13(3), 611-637 (2000) 
11. Santos, F.: Realizable but not strongly Euclidean oriented matroids. Eur. J. Combin. 22(5), 767-776 (2001)

12. Santos, F.: Triangulations of Oriented Matroids. Memoirs of the American Mathematical Society, vol. 156. American Mathematical Society, Providence (2002)

13. Santos, F.: Non-connected toric Hilbert schemes. Math. Ann. 332(3), 645-665 (2005)

14. Santos, F.: The Cayley trick and triangulations of products of simplices. In: Barvinok, A., et al. (eds.) Integer Points in Polyhedra-Geometry, Number Theory, Algebra, Optimization. Contemporary Mathematics, vol. 374, pp. 151-177. American Mathematical Society, Providence (2005)

15. Santos, F.: Geometric bistellar flips: the setting, the context and a construction. In: International Congress of Mathematicians, Vol. III, pp. 931-962. European Mathematical Society, Zürich (2006)

16. Sturmfels, B.: On the Newton polytope of the resultant. J. Algebraic Combin. 3(2), 207-236 (1994)

17. Sturmfels, B.: Gröbner Bases and Convex Polytopes. University Lecture Series, vol. 8. American Mathematical Society, Providence (1996)

18. Sturmfels, B., Ziegler, G.M.: Extension spaces of oriented matroids. Discret. Comput. Geom. 10(1), 23-45 (1993)

19. Ziegler, G.M.: Higher Bruhat orders and cyclic hyperplane arrangements. Topology 32(2), 259-279 (1993) 